\title{
Benin: Statistical Appendix
}

This Statistical Appendix paper on Benin was prepared by a staff team of the International Monetary Fund as background documentation for the periodic consultation with the member country. It is based on the information available at the time it was completed on July 1, 2002. The views expressed in this document are those of the staff team and do not necessarily reflect the views of the government of Benin or the Executive Board of the IMF.

The policy of publication of staff reports and other documents by the IMF allows for the deletion of market-sensitive information.

To assist the IMF in evaluating the publication policy, reader comments are invited and may be sent by e-mail to Publicationpolicy@imf.org.

Copies of this report are available to the public from

International Monetary Fund - Publication Services

$70019^{\text {th }}$ Street, N.W. • Washington, D.C. 20431

Telephone: (202) 623-7430 - Telefax: (202) 623-7201

E-mail: publications@imf.org Internet: http://www.imf.org

Price: $\$ 15.00$ a copy

\section{International Monetary Fund \\ Washington, D.C.}




\section{INTERNATIONAL MONETARY FUND}

\section{BENIN}

\section{Statistical Appendix}

Prepared by a staff team consisting of Mr. Ewenczyk (head), Mr. Nsengiyumva, Mrs. Nkusu, Mr. Randriamaholy (Resident Representative), and Mrs. Yontcheva (all AFR)

Approved by African Department

July 1,2002

Contents

Page

Basic Data, 1994-2001 3

Statistical Tables

1. Gross Domestic Product by Sector of Origin at Current Prices, 1994-2001.

2. Gross Domestic Product by Sector of Origin at Constant Prices 1985 Prices, 1994-2001

3. Supply and Use of Resources at Current Prices, 1994-2001

(In billions of CFA francs)

4. Supply and Use of Resources at Current Prices

1994-2001 (In percent of GDP).

5. Marketed Production and Producer Prices of Main Cash Crops, 1994/95-2000/01

6. Production and Cultivated Area of Principal Food Crops, 1994/95-2000/01

7. Production and Exports of Palm Products, 1994-2001

8. SONAPRA's Cost Structure, 1994/95-2000/01

9. Energy Supply and Consumption, 1994-2001...

10. Retail Price of Major Petroleum Products, 1994-2001

11. Structure of Prices of Water and Electricity, 1994-2000

12. Transportation Activity, 1994-2001

13. Retail Prices of Selected Products, 1991-2001.

14. Consumer Price Index in Urban Areas, 1994-2001.

15. Industrial Minimum Legal Wage, 1991-2001

16. Consolidated Government Operations, 1994-2001 
17. Central Government Revenue, 1994-2001

18. Central Government Expenditure, 1994-2001 …..............................................22

19. Central Government Investment Expenditure, 1994-2001 _...................................23

20. Operations of the Social Security Fund, 1994-2001............................................24

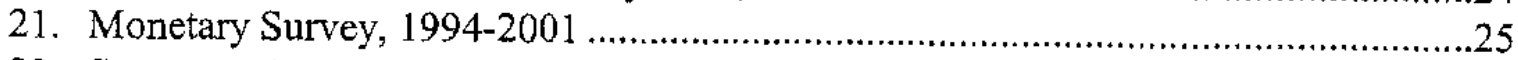

22. Summary Account of the Central Bank (BCEAO), 1994-2001 ….........................26

23. Summary Account of Commercial Banks, 1994-2001 ...........................................27

24. Net Claims on the Central Government, 1994-2001 .............................................28

25. Distribution of Credit to the Economy, 1994-2001 .............................................29

26. Central Bank Lending Rates, January 1998-December 2001 …..............................30

27. Commercial Banks' Interest Rates, December 1998-December 2001 .......................31

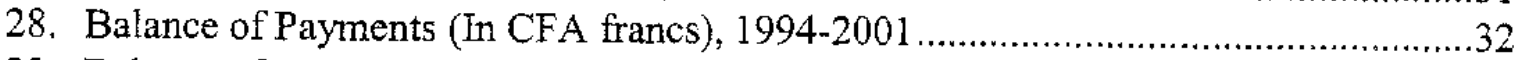

29. Balance of Payments (In U.S. dollars), 1994-2001 ...............................................33

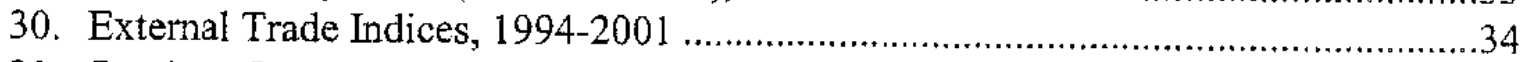

31. Services, Income, and Private Transfers, 1994-2001 ……....................................35

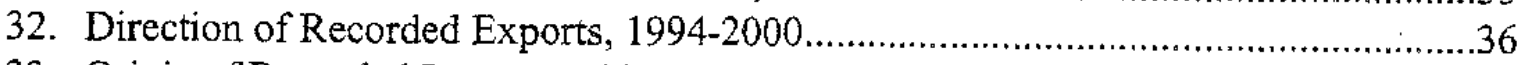

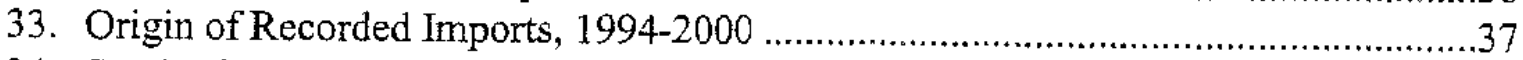

34. Stock of Public and Guaranteed External Debt, 1994-2001....................................38

35. Debt-Service Obligations on Public and Publicly Guaranteed Debt, 1994-2001 .39

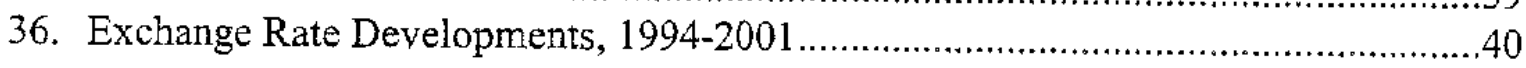

Summary of the Tax System as of April 2002 …........................................................41 
Benin: Basic Data, 1994-2001

Area, population, and GDP per capita

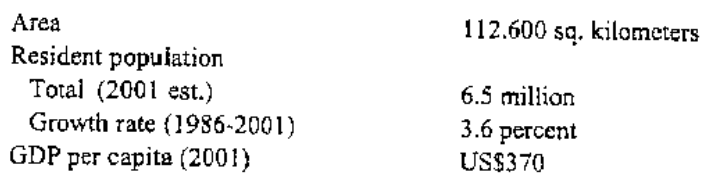

\begin{tabular}{|c|c|c|c|c|c|c|c|c|}
\hline & 1994 & 1995 & 1996 & 1997 & 1998 & 1999 & 2000 & 2001 \\
\hline National accounts at current prices & \multicolumn{8}{|c|}{ (In billions of CFA francs) } \\
\hline \multirow[t]{2}{*}{ Gross domestic product } & 831.1 & $1,002.9$ & $1,137.5$ & $1,258.8$ & $1,377.3$ & $1,469.9$ & $1,605.4$ & $1,738.6$ \\
\hline & \multicolumn{8}{|c|}{ (In percent of GDP) } \\
\hline Primary sector & 33.5 & 34.0 & 36.9 & 37.5 & 38.2 & 37.8 & 36.5 & 35.5 \\
\hline Secondary secror & 14.7 & 14.6 & 14.3 & 14.5 & 13.5 & 13.7 & 13.9 & 14.4 \\
\hline Tertiary sector & 46.8 & 45.4 & 42.7 & 41.4 & 41.4 & 40.9 & 41.7 & 42.1 \\
\hline Duties and taxes on imports & 5.1 & 6.0 & 6.2 & 6.6 & 6.9 & 7.5 & 7.9 & 7.9 \\
\hline Consumption & 92.1 & 91.5 & 92.4 & 94.4 & 93.4 & 95,2 & 94.0 & 93.5 \\
\hline Total investment & 15.8 & 19.6 & 17.1 & 18.4 & 17.0 & 17.5 & 18.9 & 19.2 \\
\hline Fixed investmetr & 15.5 & 17.2 & 16.5 & 18.4 & 17.0 & 17.5 & 18.9 & 19.2 \\
\hline Change in stocks & 0.2 & 2.4 & 0.5 & 0.0 & 0,0 & 0.0 & 0.0 & 0.0 \\
\hline Resource gap & 7.9 & 11.1 & 9.4 & 12.8 & 10.4 & 12.7 & 12.9 & 12.7 \\
\hline \multirow[t]{2}{*}{ Gross domestic savings } & 7.9 & 8.5 & 7.6 & 5.6 & 6.6 & 4.8 & 6.0 & 6.5 \\
\hline & \multicolumn{8}{|c|}{ (Percent change) } \\
\hline GDP at constant prices & 4.4 & 4.6 & 6.0 & 5.7 & 4.6 & 4.7 & 5.8 & 5.0 \\
\hline \multicolumn{9}{|l|}{ Prices } \\
\hline Implicit GDP deflator & 33.5 & 15.4 & 6.7 & 4.7 & 4.2 & 1.9 & 3.3 & 3.1 \\
\hline Consumer price index (anrual average) & 38.5 & 14.5 & 4.9 & 3.8 & 5.8 & 0.3 & 4.2 & 4.0 \\
\hline Central govemment finances & \multicolumn{8}{|c|}{ (In billions of CFA francs) } \\
\hline Total revenue & 106.4 & 149.: & 171.9 & 181.9 & 210.8 & 234.9 & 266.2 & 281.0 \\
\hline $\begin{array}{l}\text { Total expenditure } \\
\text { Or which }\end{array}$ & 164.4 & $22: .9$ & 220.4 & 234.4 & 225.2 & 258.0 & 322.5 & 353.2 \\
\hline $\begin{array}{l}\text { Of which } \\
\text { Primary expenditure }\end{array}$ & 88.3 & 126.0 & 127.9 & 142.3 & 146.6 & 175.3 & 220.7 & 257.8 \\
\hline \multicolumn{9}{|l|}{ Overall fiscal balance, } \\
\hline payment order basis, excl. grants & -58.1 & -72.7 & -48.5 & -52.5 & -14.4 & -23.1 & .56 .3 & .72 .3 \\
\hline Foreign financing & 85.3 & 83.6 & 86.3 & 63.5 & 91.1 & 80.3 & 73.7 & 119.6 \\
\hline Domestic financing & -13.4 & 6.0 & -18.2 & 3.5 & -26.0 & -35.8 & 13.9 & -49.0 \\
\hline \multirow[t]{2}{*}{ Change in external arrears } & -3.2 & 0.0 & 11.9 & 7.9 & -30.0 & .13 .3 & -14.8 & 0.0 \\
\hline & \multicolumn{8}{|c|}{ (In percent of GDP) } \\
\hline Revenue & 12.8 & 14.9 & 15.2 & 14.6 & 15.5 & 16.1 & 16.6 & 16.2 \\
\hline Primary expenditure & 10.6 & 12.6 & 11.3 & $: 1.4$ & 10.8 & 12.0 & 13.7 & 14.8 \\
\hline Govermment Investment outlays & 6.5 & 7.8 & 6.4 & 6.9 & 5.9 & 6.3 & 7.6 & 7.8 \\
\hline $\begin{array}{l}\text { Overall fiscal balance, } \\
\text { payment order basis, excl. grants }\end{array}$ & -7.0 & -7.2 & -4.3 & -4.2 & -1.1 & -1.6 & -3.5 & -4.2 \\
\hline
\end{tabular}


Benin: Basic Data, 1994-2001 (concluded)

\begin{tabular}{|c|c|c|c|c|c|c|c|c|}
\hline & 1994 & 1995 & 1996 & 1997 & 1998 & 1999 & 2000 & 2001 \\
\hline Money and credit & \multicolumn{8}{|c|}{ (In billions of CFA francs; end of period) } \\
\hline Net foreign assets & 122.7 & 144,1 & 173.1 & 215.2 & 205.4 & 306.3 & 352.7 & 479.4 \\
\hline Net domestic assets & 95.1 & 109.3 & 108.8 & 114.9 & 115.7 & 127.3 & 172.9 & 113.2 \\
\hline Credit to the government & 66.0 & 76.9 & 60.2 & 59.0 & 32.7 & -13.6 & -12.3 & -60.1 \\
\hline Credit to the economy & 40.6 & 44.2 & 65.4 & 72.0 & 101.2 & 154.6 & 194.0 & 193.5 \\
\hline Other items, net & -11.5 & -11.9 & -16.8 & -16.1 & -18.2 & -13.7 & -8.8 & -20.2 \\
\hline Money (M2) & 217.8 & 253.3 & 281.9 & 330.1 & 321.1 & 433.6 & 525.6 & 592.6 \\
\hline Balance of payments & \multicolumn{8}{|c|}{ (In billions of CFA francs) } \\
\hline $\begin{array}{c}\text { Exports, f.o.b. } \\
\text { Of which }\end{array}$ & 96.9 & 117.3 & 134.3 & 111.6 & 141.2 & 137.0 & 132.9 & 153.2 \\
\hline Cotton & 74.1 & 90.4 & 111.8 & 90.0 & 119.1 & 114.2 & 107.3 & 115.9 \\
\hline Imports, f.o.b. & -154.6 & -219.1 & -227.3 & -248.5 & -265.8 & -291.2 & -314.8 & -340.1 \\
\hline Trade balance & -57.8 & -101.8 & -92.9 & -136.9 & -124.7 & -154.2 & -181.9 & -186.9 \\
\hline Services (net) & -7.9 & -9.9 & -14.3 & -24.2 & -18.7 & -32.2 & -25.8 & -34.1 \\
\hline Income (net) & -19.5 & -22.1 & -23.5 & -17.4 & -7.9 & -9.6 & -8.7 & -15.6 \\
\hline Current transfers (net) & 63.3 & 67.9 & 83.4 & 85.7 & 72.7 & 84.9 & 87.6 & 120.6 \\
\hline Current account & -22.0 & -65.9 & -47.4 & -92.8 & .78 .6 & -111.1 & -128.7 & -116.0 \\
\hline Financial account (net) & 12.7 & .0 .6 & 28.6 & 44.3 & 41.1 & 181.3 & 148.4 & 180.8 \\
\hline Capital account & 30.8 & 37.0 & 30.7 & 49.1 & 36.4 & 40.9 & 32.3 & 31.8 \\
\hline \multirow[t]{2}{*}{ Overall balance } & 21.6 & -29.4 & 11.9 & 0.5 & -1.1 & 111.1 & 52.0 & 96.6 \\
\hline & \multicolumn{8}{|c|}{ (Percentage change) } \\
\hline Volume of exports & 43.8 & $-4,4$ & 23.7 & -18.4 & 18.9 & 25.1 & -11.0 & -2.8 \\
\hline Cotton products & 51.9 & -9.3 & 45.0 & -23.6 & 18.6 & 31.6 & -11.1 & -3.2 \\
\hline Export unit price & 113.4 & 26.6 & -7.4 & 1.8 & 6.4 & -22.4 & 9.0 & 18.6 \\
\hline Ginned cotton & 112.0 & 34.6 & -3.6 & -4.3 & 4.2 & -26.1 & 5.5 & 11.7 \\
\hline Volume of imports & -29.9 & 44.3 & 2.2 & 5.6 & 6.5 & 9.9 & 0.7 & 5.0 \\
\hline \multirow[t]{2}{*}{ Import unit price } & 111.2 & -1.8 & 1.5 & 3.5 & 0.4 & -0.3 & 7.3 & 2.8 \\
\hline & \multicolumn{8}{|c|}{ (In percent of GDP) } \\
\hline Current account & -2.6 & -6.6 & -4.2 & -7.4 & -5.8 & -7.6 & -8.0 & -6.7 \\
\hline Overall balance & 2.6 & -2.9 & 1.1 & 0.0 & -0.1 & 7.6 & 3.2 & 5.6 \\
\hline
\end{tabular}

Sources: Beninese authorities; and staff estimates. 
Table 1. Benin: Gross Domestic Product by Sector of Origin at Current Prices, 1994-2001

\begin{tabular}{|c|c|c|c|c|c|c|c|c|}
\hline & 1994 & 1995 & 1996 & 1997 & 1998 & 1999 & 2000 & 2001 \\
\hline & \multicolumn{8}{|c|}{ (In billions of CFA francs) } \\
\hline Primary sector & 278.0 & 341.3 & 419.2 & 471.8 & 526.2 & 556.3 & 586.5 & 617.7 \\
\hline Agriculture and forestry & 207.6 & 256.8 & 321.0 & 367.3 & 419.6 & 441.4 & 466.9 & 482.8 \\
\hline Livestock and fishing & 70.4 & 84.5 & 98.2 & 104.5 & 106.6 & 114.9 & 119.6 & 134.9 \\
\hline Secondary sector & 22.3 & 346.1 & 162.9 & 182.1 & 186.0 & 201.3 & 222.9 & 251.0 \\
\hline Manufacturing and handicrafts & 72.6 & 86.7 & 101.4 & 113.8 & 117.9 & 127.2 & 140.8 & 159.7 \\
\hline Construction and public works & 36.8 & 46.9 & 47.1 & 52.5 & 53.8 & 58.2 & 64.2 & 70.7 \\
\hline Mining and petroleum & 6.0 & 5.0 & 5.9 & 5.8 & 4.0 & 3.3 & 3.7 & 4.0 \\
\hline Water, gas, and electricity & 6.9 & 7.5 & 8.5 & 10.0 & 10.3 & 12.6 & 14.3 & 16.6 \\
\hline Tertiary sector & 388.6 & 454.8 & 485.4 & 521.7 & 570.6 & 601.5 & 669.2 & 732.1 \\
\hline Trade & 155.6 & 191.4 & 208.9 & 227.5 & 245.5 & 261.8 & 292.7 & 318.0 \\
\hline Transport and other services & 69.3 & 81.2 & 83.9 & 88.8 & 96.4 & 100.9 & 122.1 & 139.8 \\
\hline Public administration & 67.9 & 76.1 & 79.7 & 84.3 & 91.1 & 94.4 & 100.2 & 106.8 \\
\hline Other services & 95.8 & 106.1 & 112.9 & I21.I & 137.6 & 144.4 & 154.2 & 167.5 \\
\hline GDP at factor cost & 789.0 & 942.3 & $1,067.5$ & $!, 175.6$ & $1,282,8$ & $1,359.1$ & $1,478.6$ & $1,600.8$ \\
\hline Indirect taxes (net) & 42.1 & 60.7 & 70.0 & 83.2 & 94.5 & 110.8 & 126.8 & 137.8 \\
\hline \multirow[t]{2}{*}{ GDP at market prices } & 831.1 & $1,002.9$ & $1,137.5$ & $1,258.8$ & $1,377.3$ & $1,469.9$ & $1,605.4$ & $1,738.6$ \\
\hline & \multicolumn{8}{|c|}{ (In percent of GDP) } \\
\hline Primary sector & 33.5 & 34.0 & 36.9 & 37.5 & 38.2 & 37.8 & 36.5 & 35.5 \\
\hline Agriculture and forestry & 25.0 & 25.6 & 28.2 & 29.2 & 30.5 & 30.0 & 29.1 & 27.8 \\
\hline Livestock and fishing & 8.5 & 8.4 & 8.6 & 8.3 & 7.7 & 7.8 & 7.4 & 7.8 \\
\hline Secondary sector & 14.7 & 14.6 & 14.3 & 14.5 & 13.5 & 13.7 & 13.9 & 14,4 \\
\hline Manufacturing and handicrafts & 8.7 & 8.6 & 8.9 & 9.0 & 8.6 & 8.7 & 8.8 & 9.2 \\
\hline Construction and public works & 4.4 & 4.7 & 4.1 & 4.2 & 3.9 & 4.0 & 4.0 & 4.1 \\
\hline Mining and petroleum & 0.7 & 0.5 & 0.5 & 0.5 & 0.3 & 0.2 & 0.2 & 0.2 \\
\hline Water, gas, and electricity & 0.8 & 0.7 & 0.7 & 0.8 & 0.7 & 0.9 & 0.9 & 1.0 \\
\hline Tertiary sector & 46.8 & 45.4 & 42.7 & 41.4 & 41.4 & 40.9 & 41.7 & 42.1 \\
\hline Trade & 18.7 & 19.1 & 18.4 & 18.1 & 17.8 & 17.8 & 18.2 & 18.3 \\
\hline Transport and other services & 8.3 & 8.1 & 7.4 & 7.1 & 7.0 & 6.9 & 7.6 & 8.0 \\
\hline Public administration & 8.2 & 7.6 & 7.0 & 6.7 & 6.6 & 6.4 & 6.2 & 6.1 \\
\hline Other services & 11.5 & 10.6 & 9.9 & 9.6 & 10.0 & 9.8 & 9.6 & 9.6 \\
\hline GDP at factor cost & 94.9 & 94.0 & 93.8 & 93.4 & 93.1 & 92.5 & 92.1 & 92.1 \\
\hline Indirect taxes (net) & 5.1 & 6.0 & 6.2 & 6.6 & 6.9 & 7.5 & 7.9 & 7.9 \\
\hline GDP at market prices & 100.0 & 100.0 & 100.0 & 100.0 & 100.0 & 100.0 & 100.0 & 100.0 \\
\hline
\end{tabular}

Sources: Institut National de la Statistique et de l'Analyse Economique (INSAE); and stafí estimates. 
Table 2. Benin: Gross Domestic Product by Sector of Origin

at Constant 1985 Prices, 1994-2001

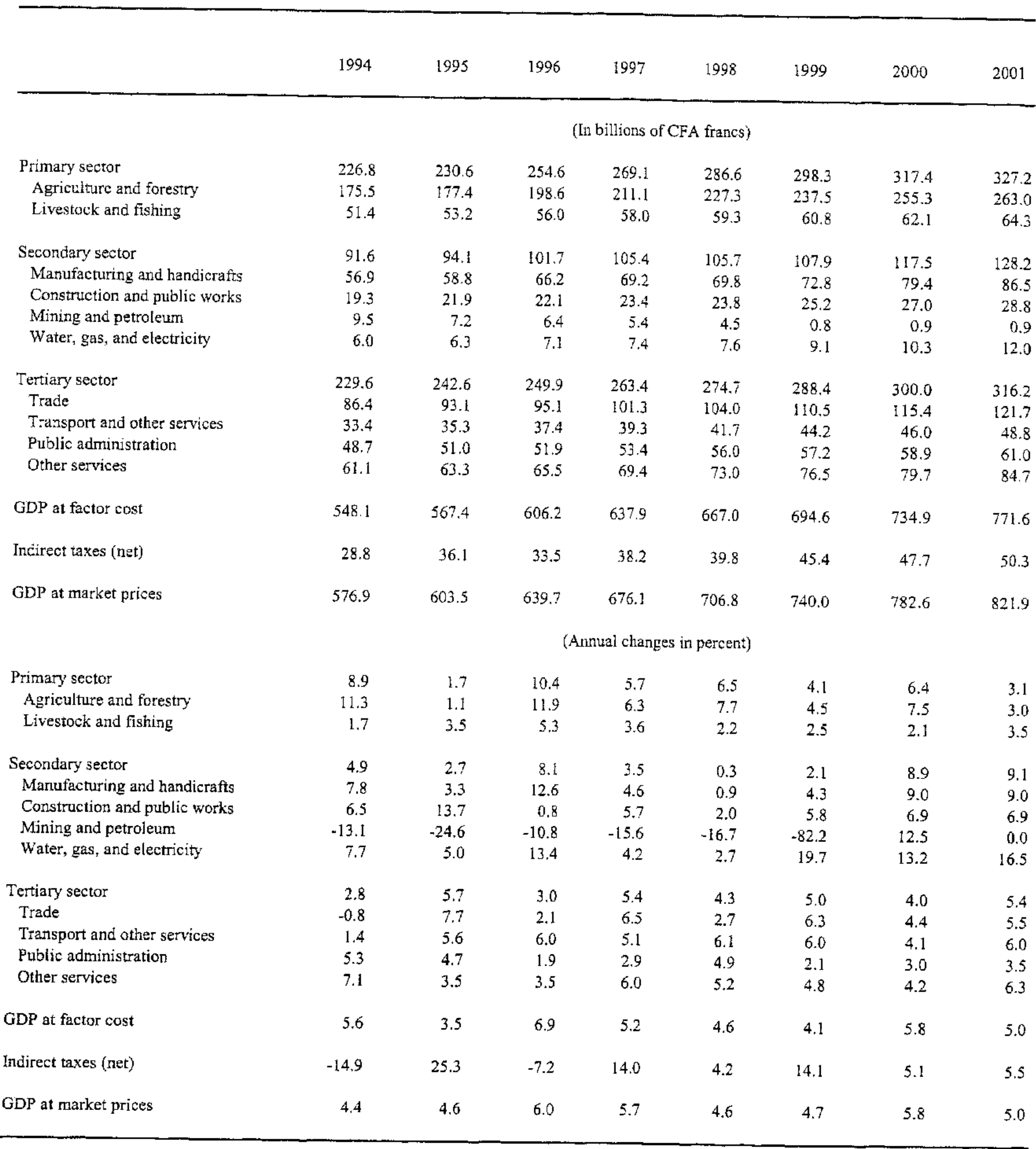

Sources: Institut National de la Statistique et de l'Analyse Economique (INSAE); and staff estimates. 
Table 3. Benin: Supply and Use of Resources at Current Prices, 1994-2001

(In billions of CFA francs)

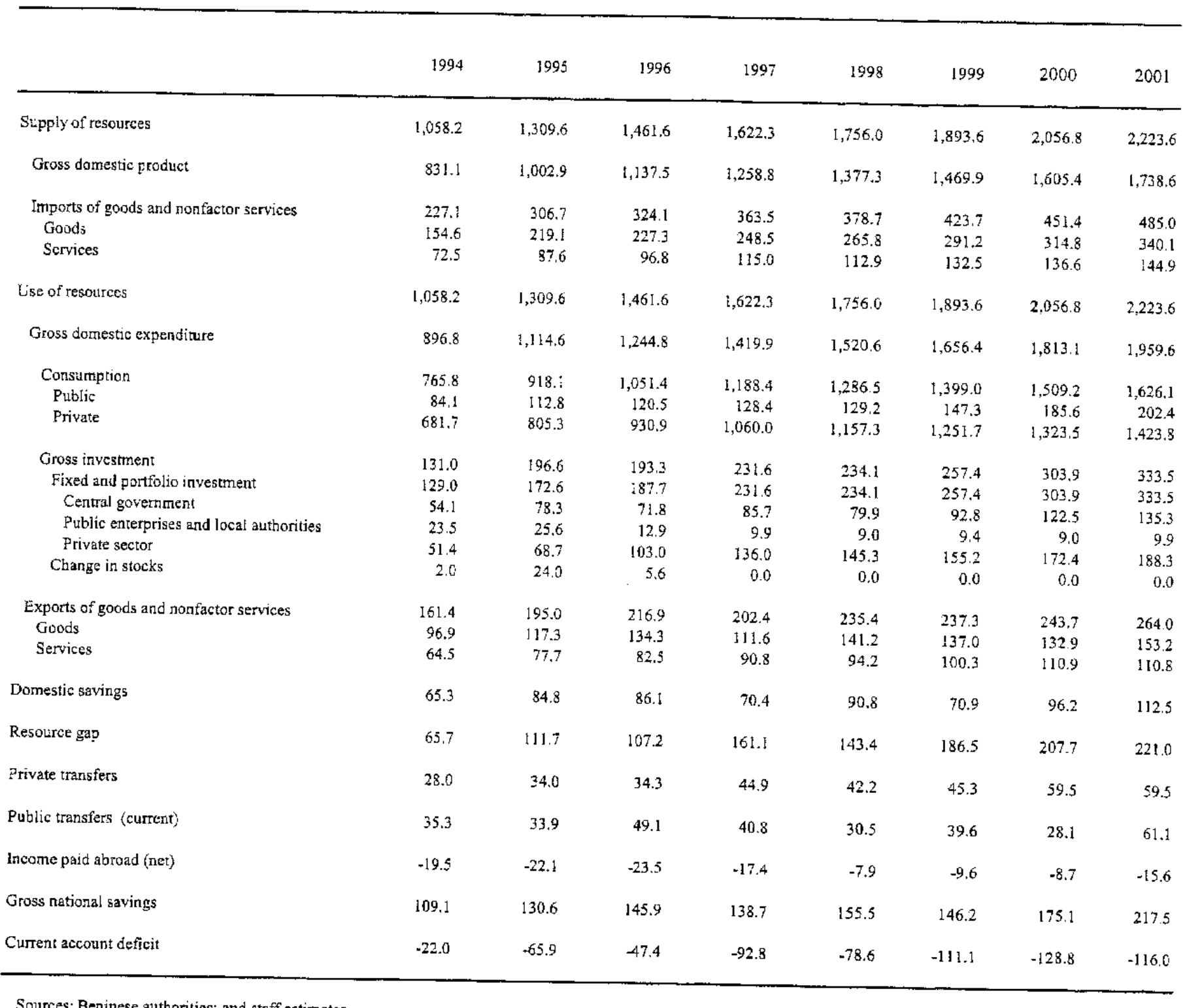

Sources: Beninese authoritics; and staff estimates. 
Table 4. Benin: Supply and Use of Resources at Current Prices, 1994-200!

(In percent of GDP)

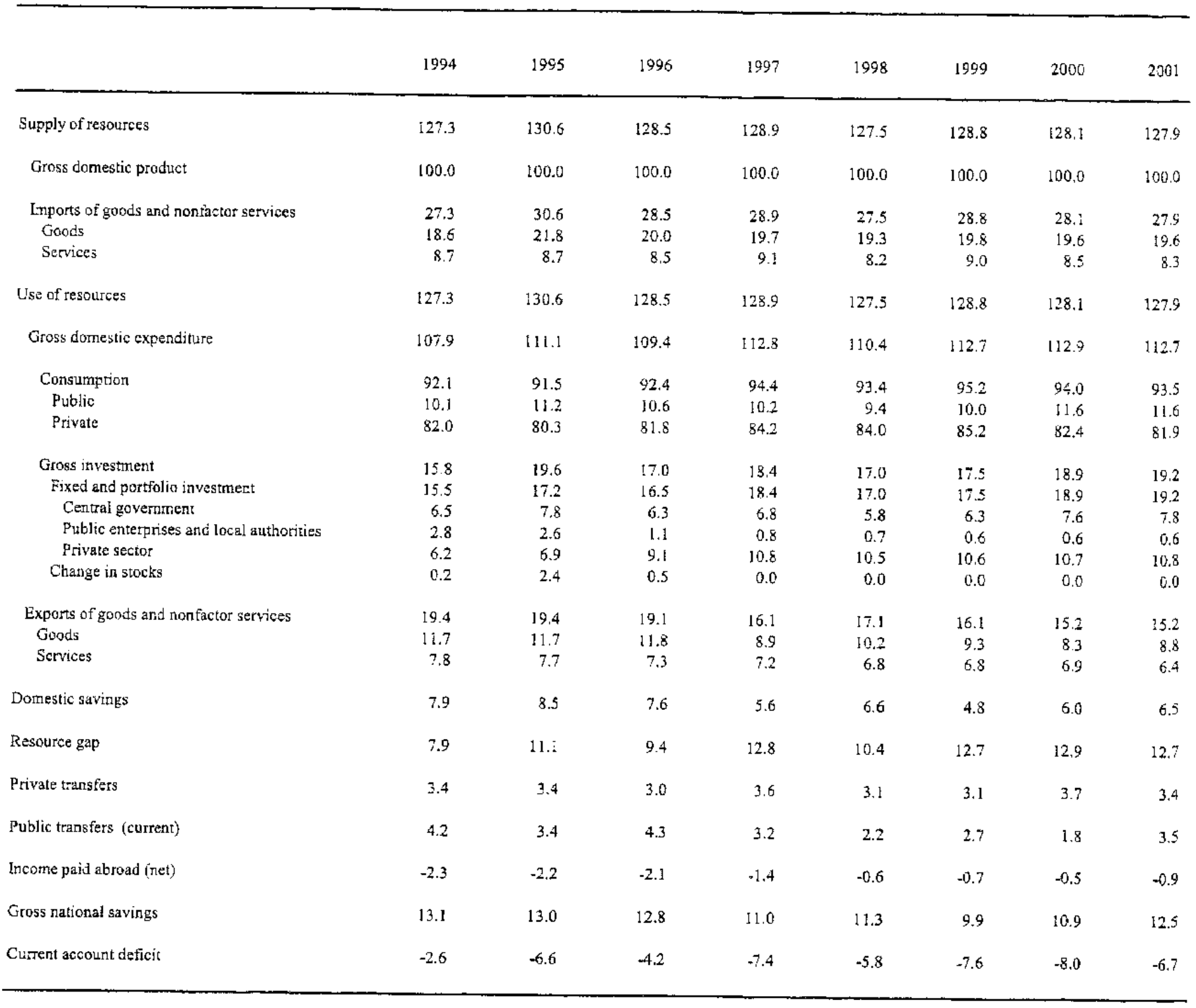

Sources: Beninese authorities; and staff estimates. 
Table 5. Benin: Marketed Production and Producer Prices of Main Cash Crops, 1994/95-2000/01 1/

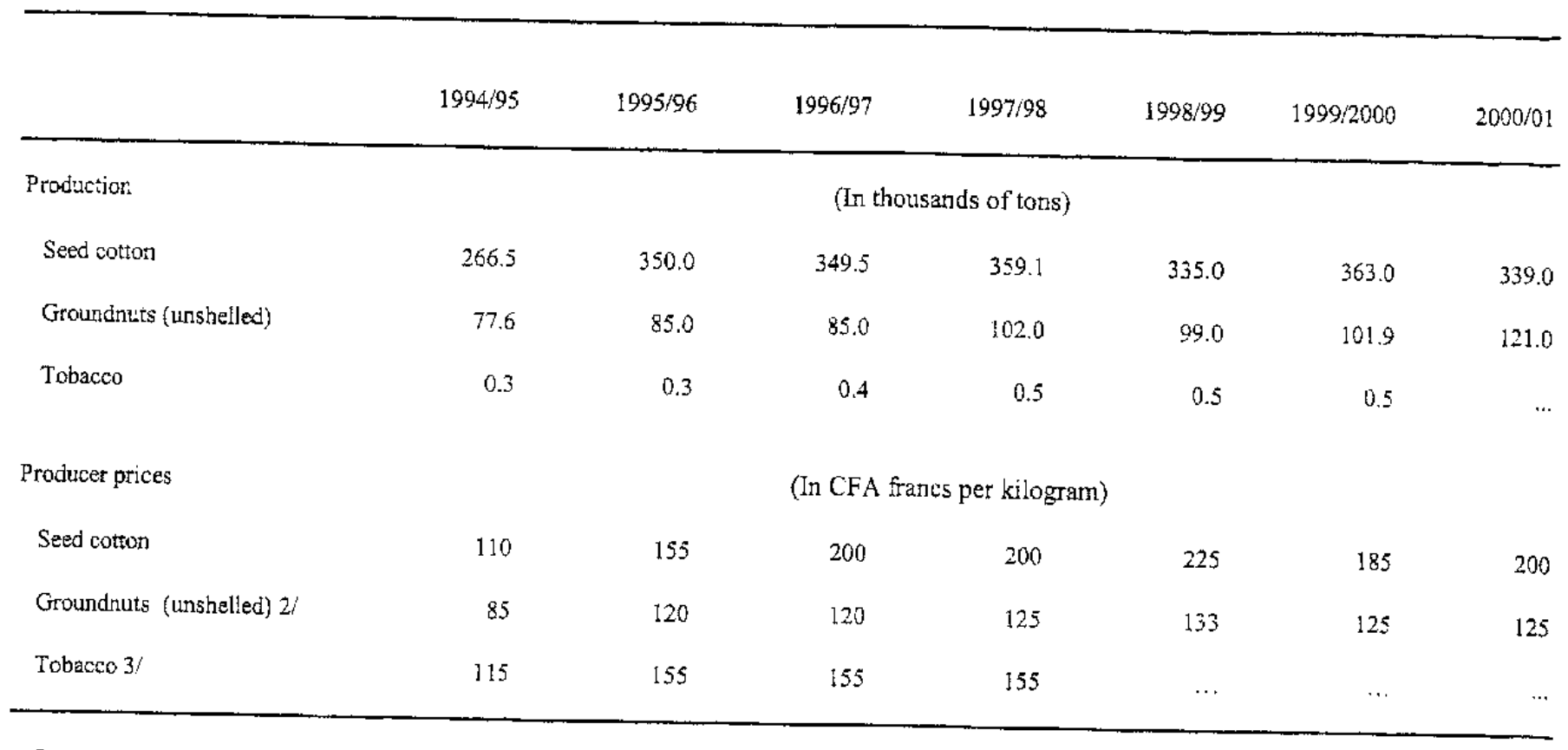
Sources: Ministry of Rural Development, Department of Planning and Research; and Sociéte Nationale pour la Promotion Agricole
(SONAPRA).

1/ Crop year: October 1-September 30.

$2 i$ Sold at the Bohicon markets.

3/ Second quality. 
Table 6. Benin: Production and Cultivated Area of Principal Food Crops, 1994/95-2000/01

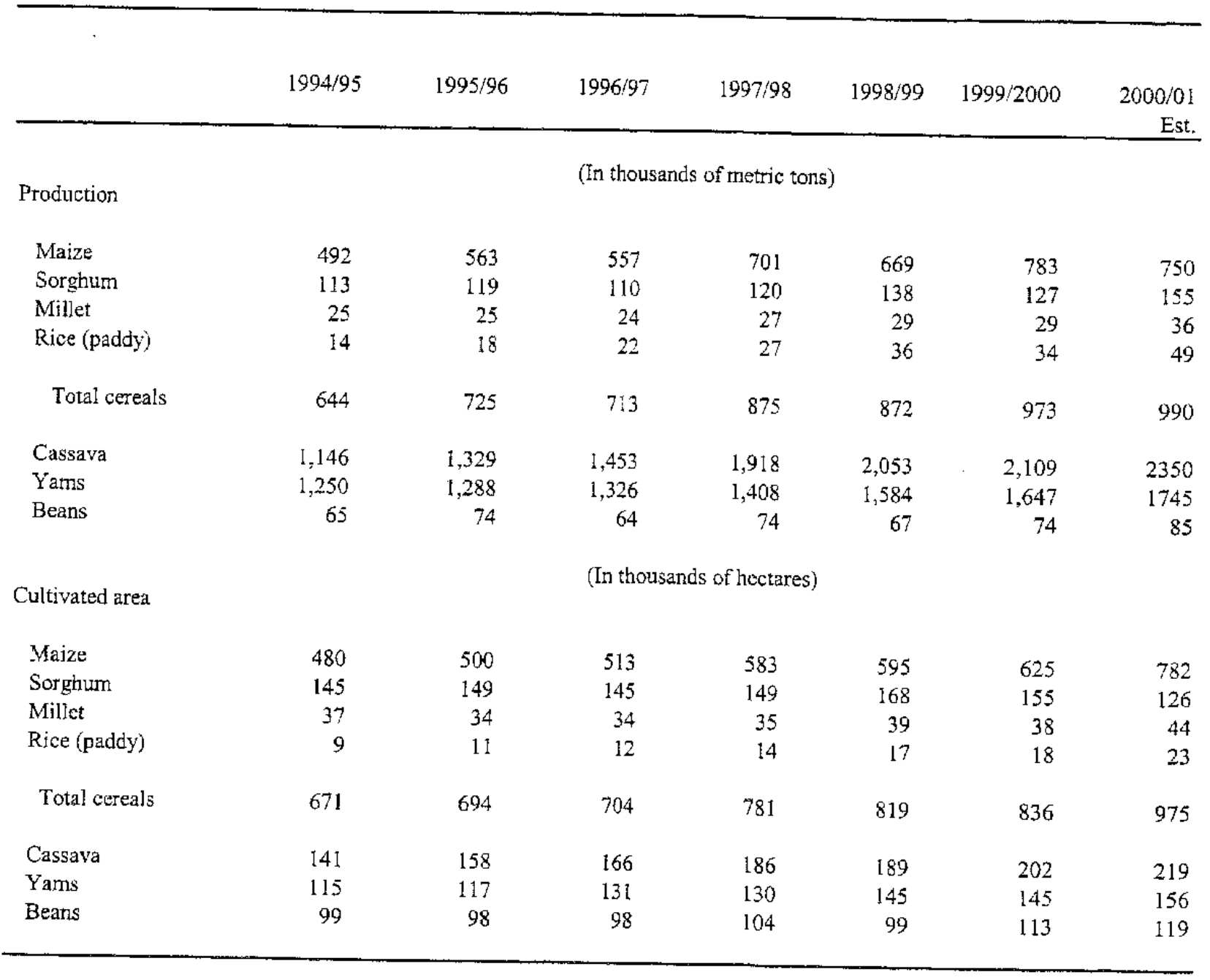

Source: Ministry of Rural Development, Department of Planning and Research. 
Table 7. Benin: Production and Exports of Palm Products, 1994-2001 1/

(In thousands of metric tons)

\begin{tabular}{|c|c|c|c|c|c|c|c|c|}
\hline & 1994 & 1995 & 1996 & 1997 & 1998 & 1999 & 2000 & $\begin{array}{r}2001 \\
\text { Est. }\end{array}$ \\
\hline \multicolumn{9}{|l|}{ Palm oil mills } \\
\hline Palm fruit processed & 54.5 & 25.7 & 35.5 & 38.1 & 41.0 & 44.0 & 61.4 & 28.9 \\
\hline Palm oil produced & 9.4 & 5.0 & 6.8 & 6.4 & 6.9 & 9.1 & 11.3 & 5.1 \\
\hline Palm kemel produced & 2.1 & 1.6 & 1.6 & 1.4 & 1.4 & 2.7 & 2.7 & 1.2 \\
\hline Palm kentel oil mills & & . & & & & & & \\
\hline Palm keme]s processed & 2.3 & 0.9 & 1.6 & 0.6 & 0.6 & 1.6 & 1.4 & 0.6 \\
\hline Palm kemel oil produced & 0.9 & 0.4 & 0.6 & 0.2 & 0.2 & 0.6 & 0.5 & 0.2 \\
\hline Paim kernel cake produced & 1.2 & 0.5 & 0.8 & 0.3 & 2.4 & 2.8 & 0.7 & 0.3 \\
\hline \multicolumn{9}{|l|}{ Export volumes } \\
\hline Paln oil & 10.5 & 8.2 & 1.1 & 0.7 & 13.5 & 7.9 & 5.3 & 2.4 \\
\hline Palm kemel oil & 2.0 & 0.5 & 0.1 & 0.1 & 0.0 & 1.6 & 0.0 & 0.0 \\
\hline Patm kernel cake & 1.5 & 1.5 & 0.7 & 0.0 & 2.5 & 2.8 & 0.0 & 0.0 \\
\hline
\end{tabular}

Sources: Société Nationale pour l'Industrie des Corps Gras (SONICOG); Central Bank of West African States (BCEAO); and staff estimates.

1/ Operations of SONICOG. 
Table 8. Benin: SONAPRA's Cost Structure, 1994/95-2000/01 1/

(In CFA francs per kilogram, unless otherwise indicated)

\begin{tabular}{|c|c|c|c|c|c|c|c|}
\hline & $1994 / 95$ & $1995 / 96$ & $1996 / 97$ & $1997 / 98$ & $1998 / 99$ & $1999 / 2000$ & $2000 / 01$ \\
\hline \multicolumn{8}{|l|}{ Seed cotton } \\
\hline Producer price of seed cotton & 134.2 & 165.0 & 200.0 & 200.0 & 221.2 & 185.0 & 200.0 \\
\hline Commission and local taxes & 5.9 & 7.1 & 7.1 & 7.1 & 7.1 & 6.4 & 10.0 \\
\hline Transport costs & 9.3 & 10.4 & 9.9 & 12.5 & 10.4 & 8.0 & 10.5 \\
\hline Purchase cost per unit of seed cotton & 149.4 & 182.5 & 217,0 & 219.6 & 238.7 & 199.4 & 220.5 \\
\hline \multicolumn{8}{|l|}{ Ginned cotton } \\
\hline Purchase costs per unit of ginned cotton & 359.6 & 452.1 & 524.5 & 523.3 & 556.0 & 468.4 & 518.0 \\
\hline Ginning costs & 34.4 & 40.5 & 44.7 & 45.8 & 47.3 & 43.6 & 54.0 \\
\hline Financial costs & 2.9 & 15.9 & 12.4 & 20.5 & 46.6 & 36.0 & 17.6 \\
\hline Transport costs & 11.7 & 13.0 & 13.9 & 14.0 & 13.0 & 15.7 & 14.0 \\
\hline Other & 113.2 & 67.3 & 105.1 & 75.7 & 145.4 & 168.4 & 156.7 \\
\hline Insurance, freight, and other export costs & 16.6 & 16.7 & 19.3 & 14.8 & 15.4 & 24.2 & 19.5 \\
\hline $\begin{array}{l}\text { Unit production costs of ginned coiton (c.i.f.) } \\
\text { Of which }\end{array}$ & \multicolumn{4}{|c|}{ Of which } & 823.7 & & 779.9 \\
\hline Variable costs & 471.4 & 548.1 & 646.6 & 619.7 & 699.6 & 639.4 & 689.1 \\
\hline Fixed costs & 67.1 & 57,3 & 73.3 & 74.4 & 124.1 & 116.9 & 90.8 \\
\hline \multicolumn{8}{|l|}{ Cottonseed } \\
\hline Transport costs & 8.4 & 9.3 & 9.8 & 10.0 & 17.9 & 12.1 & 11.0 \\
\hline Other production costs & 1.3 & 0.9 & 1.3 & 1.0 & 0.6 & 0.6 & 1.6 \\
\hline Insurance, freight, and other export costs & 3.2 & 4.6 & 4.6 & 6.2 & 5.4 & 8.9 & 5.3 \\
\hline Lnit production costs of cottonseed (c.if.) & 12.9 & 14.8 & 15.7 & 17.2 & 23.9 & 21.6 & 17.9 \\
\hline \multicolumn{8}{|l|}{ Sales prices } \\
\hline Ginned cotton & 895.3 & 800.5 & 830.4 & 810.0 & 664.0 & 639.0 & $\ldots$ \\
\hline Cottonseed & 32.3 & 30.8 & 37.6 & 66.0 & 48.8 & 45.7 & 37.0 \\
\hline \multicolumn{8}{|l|}{ Profit per unit } \\
\hline Ginned cotton & 356.9 & 195.1 & 110.5 & 115.9 & -159.7 & -117.3 & $\ldots$ \\
\hline Cottonseed & 19.4 & 16.0 & 21.9 & 48.9 & 24.9 & 24.0 & 19.1 \\
\hline \multicolumn{8}{|l|}{ Memorandum items: } \\
\hline Total profits (in billions of CFA francs) & 37.4 & 24.2 & 16.5 & 20.6 & -11.9 & -10.0 & $\cdots$ \\
\hline Contribution to government revenue (in percent) & 16.0 & 12.1 & 7.1 & 5.7 & $\ldots$ & $\ldots$ & $\ldots$ \\
\hline Unit production cost of ginned cotton (f.o.b.) & 521.7 & 588.8 & 700.6 & 679.3 & 808.2 & 732.1 & 760.3 \\
\hline
\end{tabular}

Source: Société Nationale pour la Promotion Agricole (SONAPRA); and staff estimates.

1/ Crop year: October 1 - September 30. 
Table 9. Benin: Energy Supply and Consumption, 1994-2001

\begin{tabular}{|c|c|c|c|c|c|c|c|c|}
\hline & 1994 & 1995 & 1996 & 1997 & 1998 & 1999 & 2000 & 2001 \\
\hline & \multicolumn{8}{|c|}{ (In millions of barrels) } \\
\hline Production of crude oil & 0.9 & 0.7 & 0.6 & 0.5 & 0.3 & 0.0 & 0.0 & 0.0 \\
\hline Official imports and sales & \multicolumn{8}{|c|}{ (In metric tons) } \\
\hline Aviation fuel & 17,066 & 13,927 & 20,804 & 16,234 & 17,365 & 6,198 & $\ldots$ & $\ldots$ \\
\hline Gasoline & 69,007 & 59,678 & 73,211 & 126,882 & 149,881 & 167,384 & $\ldots$ & $\ldots$ \\
\hline Regular & 43,279 & 37,053 & 44,131 & 88,476 & 114,558 & 136,625 & $\ldots$ & $\ldots$ \\
\hline Premiurz & 25,728 & 22,625 & 29,080 & 38,406 & 35,323 & 30,759 & $\ldots$ & $\ldots$ \\
\hline Diesel fuel & 66,903 & 67,926 & 79,045 & 78,945 & 96,360 & 103,955 & $\cdots$ & $\ldots$ \\
\hline Fuel oil and light distillates & 26,218 & 9,028 & 15,169 & 19,926 & 9,839 & $\ldots$ & $\ldots$ & $\ldots$ \\
\hline Kerosene & 45,522 & 51,639 & 56,470 & 66,109 & 77,255 & 96,365 & $\ldots$ & $\ldots$ \\
\hline Other & 4,877 & 4,719 & 6,905 & 6,510 & 5,668 & 3,921 & $\ldots$ & $\ldots$ \\
\hline $\begin{array}{l}\text { Sales of petroleum products l/ } \\
\text { Of which }\end{array}$ & 229,593 & 206,917 & 251,604 & 314,606 & 356,368 & 377,823 & $\cdots$ & $\ldots$ \\
\hline Local network sales & 146,387 & 150,932 & 178,411 & 240,556 & 290,769 & 373,791 & $\cdots$ & $\ldots$ \\
\hline Direct sales to enterprises & 74,400 & 54.789 & 71,525 & 72,210 & 64,458 & $\ldots$ & $\ldots$ & $\cdots$ \\
\hline Exports & 8,722 & 996 & 1,668 & 1,840 & 1,141 & 4,032 & $\cdots$ & $\cdots$ \\
\hline Imports of petroleum products & 262,326 & 238,005 & 241,043 & 309,417 & 352,298 & 310,250 & $\cdots$ & $\ldots$ \\
\hline $\begin{array}{l}\text { Estimated unrecorded imports of } \\
\text { perroleum products } 2 /\end{array}$ & 13,395 & 48,333 & 17,473 & 10,465 & 8,187 & 149,864 & $\ldots$ & $\ldots$ \\
\hline $\begin{array}{l}\text { Estimated consumption of } \\
\text { petroleum products } 2 / 3 /\end{array}$ & 242,988 & 255,250 & 269,077 & 325,071 & 364,555 & 527,687 & $\cdots$ & $\ldots$ \\
\hline Electricity & \multicolumn{8}{|c|}{ (In thousands of kilowatt-hours) } \\
\hline $\begin{array}{l}\text { Production available } \\
\text { Of which }\end{array}$ & 262,900 & 289,600 & 310,826 & 332,338 & 313,416 & 369,180 & 428,143 & 512,046 \\
\hline Imported from Ghana & 210,200 & 256,400 & 264,138 & 282.138 & 255,461 & 325,360 & 374,275 & 456,158 \\
\hline Consumption & 225,000 & 250,200 & 269,442 & 290,130 & 269,161 & 316,653 & 367,391 & 413,587 \\
\hline
\end{tabular}
Sources: Société Nationale de Commetcialisation des Produits Pétroliers (SONACOP); Société Béninoise d'Ejectricité etd'Eau (SBEE); Sémé oil project;
DGID (Ministry of Economy and Finance); and staff estimates.

1/ Through official SONACOP distribution channeis. Following SONACOP's privatization, post-1999 data are provided by the Ministry of Economy and Finance (DGD, Direction des Grandes Entreprises).

2/Staff estimates.

3/ Sales of petroleum products plus estimated unrecorded inports of petroleum products. 
Table 10. Benin: Retail Price of Major Petroleum Products, 1994-2001

(In CFA francs per liter)

\begin{tabular}{lcccccccc}
\hline & 1994 & 1995 & 1996 & 1997 & 1998 & 1999 & 2000 & 2001 \\
\hline Premium gasoline & 175 & 175 & 175 & 200 & 220 & 220 & 350 & 320 \\
Regular gasoline & 170 & 170 & 170 & 175 & 195 & 195 & 325 & 295 \\
Kerosene & 100 & 100 & 100 & 125 & 125 & 125 & 210 & 190 \\
Diesel & 135 & 135 & 135 & 170 & 175 & 175 & 285 & 275 \\
\hline
\end{tabular}
Sources: Société Nationale de Commercialisation des Produits Pétroliers (SONACOP); and Ministry of
Finance and Economy. 
Table 11. Benin: Structure of Prices of Water and Electricity, 1994-2000

(In millions of CFA francs, unless otherwise indicated)

\begin{tabular}{|c|c|c|c|c|c|c|c|}
\hline & 1994 & 1995 & 1996 & $1997 \mathrm{~K}$ & 1998 & 1999 & 2000 \\
\hline \multicolumn{8}{|l|}{ Water } \\
\hline $\begin{array}{l}\text { Production cost } \\
\text { Of which }\end{array}$ & 1,307 & 1,379 & 1,589 & 1,700 & 940 & 1,961 & 2,062 \\
\hline Salary & 166 & 179 & 208 & 314 & 85 & 238 & 273 \\
\hline Marketing cost & 807 & 840 & 1,293 & 751 & 704 & 1,136 & 994 \\
\hline Indirect operating costs & 281 & 305 & 493 & 455 & 274 & 398 & 680 \\
\hline Business expenses & 2,028 & 2,548 & 2,554 & 2,477 & 728 & 2,841 & 3,180 \\
\hline Total cost & 4,423 & 5,071 & 5,928 & 5,383 & 2,646 & 6,335 & 6,926 \\
\hline Unit cost (in CFA francs per cubic meter) & 354 & 380 & 369 & 354 & 324 & 351 & 359 \\
\hline Unit selling price (in CFA francs per cubic meter) & 187 & 191 & 190 & 276 & 253 & 234 & 203 \\
\hline \multicolumn{8}{|l|}{ Electricity } \\
\hline $\begin{array}{l}\text { Production cost } \\
\text { of which }\end{array}$ & 10,606 & 11,752 & $12,63]$ & 13,075 & 6,469 & 16,044 & 19,106 \\
\hline Salary & 187 & 206 & 306 & 274 & 186 & 309 & 352 \\
\hline Marketing cost & 1,589 & 1,383 & 1,454 & 1,107 & 751 & 1,579 & 427 \\
\hline Indirect operating costs & 761 & 1,097 & 1,406 & 828 & 502 & 1,109 & 1,086 \\
\hline Business expenses & 4,960 & 5,130 & 1,559 & 1,238 & 1,255 & 4,209 & 6,395 \\
\hline Total cost & 17,916 & 19,363 & 17,049 & 16,248 & 8,977 & 23,023 & 27,217 \\
\hline Unit cost (in CFA francs per kilowatt-hour) & 79 & 78 & 64 & 56 & 68 & 73 & 75 \\
\hline Unit selling price (in CFA francs per kilowatt-hour) & 63 & 66 & 62 & 67 & 72 & 70 & 70 \\
\hline
\end{tabular}

Source: Société Béninoise d'Electricité et d'Eau (SBEE).

1/ Prices adjusted on January 1, 1997. 
Table 12. Benin: Transportation Activity, 1994-2001

\begin{tabular}{|c|c|c|c|c|c|c|c|c|}
\hline & 1994 & 1995 & 1996 & 1997 & 1998 & 1999 & 2000 & 2001 \\
\hline Port traffic & \multicolumn{8}{|c|}{ (In thousands of metric tons) } \\
\hline $\begin{array}{l}\text { Loading } \\
\text { Of which }\end{array}$ & 373.5 & 338.5 & 423.9 & 370.3 & 379.0 & 360.4 & 398.7 & 384.1 \\
\hline Palm procucts & 12.5 & 0.5 & 0.4 & 2.0 & 6.0 & $\ldots$ & $\ldots$ & \\
\hline Cotton products & 198.7 & 196.0 & 300.5 & 230.3 & 290.1 & 264.5 & 273.3 & 203.4 \\
\hline Transit & 5.0 & 4.5 & 5.7 & 6.6 & 6.8 & 5.3 & 4.9 & 6.5 \\
\hline $\begin{array}{l}\text { Unloading } \\
\text { Of which }\end{array}$ & $1,611.2$ & $1,738.4$ & $1,795.8$ & $1,877,9$ & $2,004.6$ & $2,236.2$ & $2,674.8$ & $2,941.5$ \\
\hline Petroleum products & 349.3 & 292.8 & 297.7 & 331.5 & 434.0 & 508.6 & 423.0 & 309.2 \\
\hline Transit & 391.6 & 369.4 & 454.4 & 488.7 & 588.0 & 507.2 & 839.6 & 984.9 \\
\hline Total & I,984.7 & $2,076.9$ & $2,219.7$ & 2.248 .2 & $2,383.6$ & $2,596.6$ & $3,073.5$ & $3,325.6$ \\
\hline Transit & 396.6 & 373.9 & 460.1 & 495.3 & 594.8 & 512.5 & 844.5 & 991.4 \\
\hline Niger & 277.2 & 223.6 & 270.7 & 340.0 & 414.8 & 346.5 & 419.7 & 507.6 \\
\hline Nigeria & 61.9 & 80.8 & 94.9 & 108.0 & 115.4 & 118.6 & 332.2 & 402.4 \\
\hline Burkina Faso & 33.7 & 20.8 & 38.6 & 2.5 & 22.5 & 17.6 & 5.2 & 5.2 \\
\hline Togo & 11.2 & 21.2 & 25.9 & 18.5 & 13.9 & 5.8 & 2.8 & 5.0 \\
\hline Malj & 12.6 & 27.5 & 30.0 & 26.3 & 28.2 & 22.9 & 44.0 & 47.4 \\
\hline Rail traffic & \multicolumn{8}{|c|}{ (In units indicated) } \\
\hline Passengers (in millions per kilomete:) & 107.0 & 116.0 & 117.0 & 121.8 & 112.0 & 82,2 & 156.6 & $\cdots$ \\
\hline Freight (in millions of tons per kilometer) & 253.0 & 388.4 & 269.7 & 311.3 & 218.7 & 269.0 & 153.2 & $\cdots$ \\
\hline Upfreight (in thousands of metric tons) & 268.5 & 211.0 & 189.4 & 226.8 & 198.6 & 262.4 & 154.8 & $\ldots$ \\
\hline General merchandjse & 175.5 & 126.0 & 119.6 & 169.6 & 169.4 & 226.0 & 132.6 & $\ldots$ \\
\hline To Benin & 20.5 & 10.0 & 7.7 & 13.4 & 15.8 & 10.2 & 6.4 & $\ldots$ \\
\hline To Niger & 155.0 & 116.0 & 111.9 & 156.2 & 153.7 & 215.8 & 126.2 & $\cdots$ \\
\hline Hydrocarbons & 93.0 & 85.0 & 69.8 & 57.3 & 29.2 & 36.4 & 22.2 & $\ldots$ \\
\hline To Benin & 39.0 & 37.0 & 29.1 & 30.5 & 10.2 & 36.4 & 22.2 & $\cdots$ \\
\hline To Niger & 54.0 & 48.0 & 40.7 & 26.7 & 18.9 & 0.0 & 0.0 & $\ldots$ \\
\hline ownfreight (in thousands of metric tons) & 119.0 & 177.0 & 80.1 & 81.5 & 141.5 & 124.0 & 117,4 & $\cdots$ \\
\hline From Benin & 111.0 & 163.0 & 74.6 & 74.8 & 135.5 & 117.6 & 113.0 & $\cdots$ \\
\hline From Niger & 8.0 & 14.0 & 5.5 & 6.7 & 6.0 & 6.4 & 4.4 & $\cdots$ \\
\hline
\end{tabular}

Sources: Ministry of Transportation, Directorate of the Port of Cotonou; Bénin-Niger Railway (OCBN); and staff estimates. 
Table 13. Benin: Retail Prices of Selected Products, 1991-2001 1/

\begin{tabular}{|c|c|c|c|c|c|c|c|}
\hline $\begin{array}{l}\text { Lump } \\
\text { Sugar }\end{array}$ & $\begin{array}{l}\text { Imported } \\
\text { Rice }\end{array}$ & Bread 2/ & Maize & $\begin{array}{c}\text { Cassava } \\
\text { Flour }\end{array}$ & Beans & $\begin{array}{l}\text { Groundnut } \\
\text { Oit }\end{array}$ & $\begin{array}{c}\text { Palm } \\
\text { Oil }\end{array}$ \\
\hline
\end{tabular}

\begin{tabular}{|c|c|c|c|c|c|c|c|c|}
\hline Dec. 1991 & 300 & 300 & 210 & 100 & 99 & 269 & 400 & 475 \\
\hline Dec. 1992 & 251 & 288 & 210 & 50 & 102 & 219 & 375 & 276 \\
\hline Dec. 1993 & 240 & 294 & 210 & 50 & 102 & 158 & 382 & 338 \\
\hline Dec. 1994 & 418 & $\ldots$ & 350 & 101 & $\ldots$ & 325 & 800 & 500 \\
\hline Dec. 1995 & 450 & 312 & 350 & 144 & 126 & 306 & 700 & 450 \\
\hline Dec. 1996 & 460 & 334 & 385 & 155 & 192 & 368 & 733 & 387 \\
\hline Dec. 1997 & 545 & 394 & 385 & 137 & 200 & 373 & 768 & 500 \\
\hline Dec. 1998 & 495 & 419 & 385 & 150 & 197 & 475 & 805 & 478 \\
\hline Dec. 1999 & 456 & 395 & 470 & 129 & 244 & 436 & 805 & 525 \\
\hline Dec. 2000 & 333 & 410 & $\ldots$ & 154 & 262 & 400 & 780 & 480 \\
\hline \multirow[t]{2}{*}{ Dec. 2001} & 265 & 375 & $\cdots$ & 150 & 370 & 459 & 780 & 530 \\
\hline & \multicolumn{8}{|c|}{ (Monthly average) } \\
\hline 1991 & 302 & 301 & 210 & 125 & 144 & 270 & 421 & 507 \\
\hline 1992 & 251 & 302 & 210 & 126 & 118 & 235 & 386 & 398 \\
\hline 1993 & 249 & 287 & 210 & 98 & 105 & 210 & 386 & 336 \\
\hline$[994$ & 442 & $\ldots$ & 320 & 90 & $\ldots$ & 313 & 604 & 329 \\
\hline 1995 & 472 & 365 & 350 & 119 & 123 & 309 & 759 & 494 \\
\hline 1996 & 469 & 298 & 352 & 159 & 165 & 466 & 725 & 405 \\
\hline 1997 & 497 & 370 & 385 & 167 & 204 & 422 & 742 & 393 \\
\hline 1998 & 717 & 380 & 385 & 154 & 191 & 431 & 792 & 453 \\
\hline 1999 & 468 & 406 & 470 & 134 & 195 & 453 & 807 & 518 \\
\hline 2000 & 266 & 406 & $\ldots$ & 150 & 270 & 441 & 796 & 459 \\
\hline 2001 & $\cdots$ & $\ldots$ & $\ldots$ & $\ldots$ & $\ldots$ & $\ldots$ & $\ldots$ & $\ldots$ \\
\hline 1991 & $-12,0$ & 3.5 & -4.5 & -9.4 & -23.0 & -15.6 & -20.1 & -25.3 \\
\hline 1992 & -20.3 & 0.3 & 0.0 & 0.8 & -18.1 & -13.0 & -8.3 & -21.5 \\
\hline$[993$ & -1.9 & -5.0 & 0.0 & -22.2 & -11.0 & -8.1 & 0.0 & -15.6 \\
\hline 1994 & 11.5 & $\ldots$ & 55.2 & -8.2 & $\ldots$ & 49.0 & 56.5 & -2.1 \\
\hline 1995 & 6.8 & $\ldots$ & 9.4 & 32.2 & $\ldots$ & -1.3 & 25.7 & 50.2 \\
\hline 1996 & -0.6 & 22.5 & 0.6 & 33.6 & 34.1 & 50.8 & -4.5 & -18.0 \\
\hline 1997 & 44.3 & 2.2 & 0.0 & -17.8 & .5 .9 & -4.7 & 6.6 & 29.7 \\
\hline 998 & -9.2 & 6.3 & 0.0 & 9.5 & -1.5 & 27.3 & 4.8 & -4.4 \\
\hline 999 & -20.7 & 6.8 & 22.1 & -13.0 & 2.1 & 5.1 & 1.9 & 14.3 \\
\hline 000 & -43.2 & 0.0 & $\ldots$ & 11.9 & 38.5 & -2.6 & -1.4 & -11.4 \\
\hline
\end{tabular}

Sources: Central Bank of West African States (BCEAO); and Institit National de la Statistique et l'Analyse Economique (WNSAE).

1/ Actual market prices.

2/ Industrially processed. 
Table 14. Benin: Consumer Price Index in Urban Areas, 1994-2001 1/

(Index, December 1991=100, unless otherwise indicated)

\begin{tabular}{|c|c|c|c|c|c|c|c|c|c|}
\hline & 1994 & 1995 & 1996 & 1997 & $19972 /$ & 1998 & 1999 & 2000 & 2001 \\
\hline January & 125.2 & 161.8 & 172.4 & 176.4 & 176.4 & 184.8 & 191.8 & 187.9 & 201.8 \\
\hline February & 133.1 & 161.1 & 168.0 & 177.5 & 175.8 & 185.2 & 189.3 & 189.6 & 199.7 \\
\hline March & 139.0 & 163.0 & 169.0 & 179.1 & 177.9 & 190.7 & 187.3 & 193.8 & 203.0 \\
\hline April & 145.4 & 165.3 & $: 73.3$ & 184.0 & 181.2 & 191.7 & 193.0 & 195.6 & 205.4 \\
\hline May & 144.0 & 168.4 & 173.9 & 182.6 & 180.7 & 192.4 & 193.6 & 197.6 & 208.9 \\
\hline June & 142.2 & 164.1 & 175.2 & 181.8 & 179.8 & 194.7 & 191.1 & 202.8 & 207.8 \\
\hline July & 144.3 & 164.5 & 177.2 & 180.5 & $\$ 79.5$ & 190.8 & $: 90.9$ & 201.1 & 208.5 \\
\hline August & 142.9 & 167.2 & 175.4 & 178.7 & 178.6 & 188.4 & 191.9 & 200.1 & 206.5 \\
\hline September & 149.8 & 167.6 & 174.9 & 177.0 & 177.2 & 186.8 & 189.8 & 198.9 & 205.8 \\
\hline October & 1.52 .4 & 170.3 & 172.5 & 180.0 & 178.4 & 185.7 & 188.3 & 202.0 & 206.1 \\
\hline November & 156.2 & 167.4 & 174.8 & 184.1 & 181.1 & $\therefore 89.0$ & 187.4 & 202.5 & 207.8 \\
\hline Decemier & 162.4 & 167.5 & 179.1 & 183.4 & 183.4 & 193.8 & 187.5 & 205.9 & 210.8 \\
\hline Average & 144.7 & 165.7 & 173.8 & 180.4 & 179.2 & 189.5 & 190.2 & 198.2 & 206.0 \\
\hline Changes in percen & 38.5 & 14.5 & 4.9 & 3.8 & 0.6 & 5.8 & 0.3 & 4.2 & 4.0 \\
\hline
\end{tabular}

Source: Institut National de la Statistique et de 1'Analyse Economique (INSAE).

1/ Index is jased on data covering tive major cities.

2/ Harmonized consumer price index of the West African Economic and Monetary Union based on a 1996 basket covering Cotonou. 
Table 15. Benin: Industrial Minimum Legal Wage, 1991-2001 (In CFA francs per hour)

\begin{tabular}{lc}
\hline Date & Legal wage \\
\hline January 1991 & 81.21 \\
January 1992 & 81.21 \\
January 1993 & 81.21 \\
May 1994 & 117.12 \\
January 1995 & 117.12 \\
January 1996 & 117.12 \\
April 1997 & 126.49 \\
January 1998 & 126.49 \\
June 1999 & 126.49 \\
March 2000 & 144.23 \\
December 2000 & 144.23 \\
\hline December 2001 & \\
\hline
\end{tabular}

Sources: Central Bank of West African States (BCEAO); and Ministry of Labor and Social Affairs. 
Table 16. Benin: Consolidated Govemment Operations, 1994-2001

\begin{tabular}{|c|c|c|c|c|c|c|c|c|}
\hline & 1994 & 1995 & 1996 & 1997 & 1998 & 1999 & 2000 & 2001 \\
\hline & \multicolumn{8}{|c|}{ (It biltions of CFA francs) } \\
\hline Total revenue & 106.4 & 149.1 & 171.9 & 181.9 & 210.8 & 234.9 & 266.2 & 281.0 \\
\hline $\begin{array}{l}\text { Tax revenue } \\
\text { Tax on international trade } 1 / \\
\text { Direct and indirect kaxes } \\
\text { Non:ax reverue }\end{array}$ & $\begin{array}{l}91.8 \\
42.1 \\
49.5 \\
14.5\end{array}$ & $\begin{array}{r}123.0 \\
60.3 \\
62.7 \\
26.1\end{array}$ & $\begin{array}{r}142.6 \\
70.3 \\
72.3 \\
29.3\end{array}$ & $\begin{array}{r}158.8 \\
82.7 \\
76.1 \\
23.1\end{array}$ & $\begin{array}{l}: 82.6 \\
91.3 \\
91.3 \\
28.2\end{array}$ & $\begin{array}{r}200.8 \\
106.8 \\
94.0 \\
34.0\end{array}$ & $\begin{array}{r}234.1 \\
126.7 \\
107.4 \\
32.1\end{array}$ & $\begin{array}{r}247.1 \\
133.2 \\
113.9 \\
33.9\end{array}$ \\
\hline Total expenditure & 164.4 & 2218 & 220.4 & 2344 & 225.2 & 258.0 & 322.5 & 353.2 \\
\hline $\begin{array}{l}\text { Wageg } \\
\text { Pensicns and scinolarships } \\
\text { Curtent transfers } \\
\text { Other experditure } \\
\text { Investment } \\
\text { Budgetary contrlbution } \\
\text { Financed from absoad }\end{array}$ & $\begin{array}{r}45.7 \\
10.4 \\
8.2 \\
19.7 \\
54.2 \\
4.3 \\
49.9\end{array}$ & $\begin{array}{r}53.9 \\
11.5 \\
8.7 \\
38.7 \\
78.2 \\
11.2 \\
65.0\end{array}$ & $\begin{array}{r}58.2 \\
16.9 \\
8.7 \\
36.7 \\
71.8 \\
7.3 \\
64.5\end{array}$ & $\begin{array}{r}62.0 \\
16.2 \\
8.9 \\
4 i .2 \\
85.7 \\
13.9 \\
71.8\end{array}$ & $\begin{array}{r}64.6 \\
16.8 \\
7.3 \\
40.5 \\
79.9 \\
17.4 \\
62.5\end{array}$ & $\begin{array}{l}66.2 \\
18.3 \\
20.5 \\
42.4 \\
92.8 \\
28.0 \\
64.8\end{array}$ & $\begin{array}{r}74.8 \\
19.3 \\
26.8 \\
64.7 \\
122.5 \\
35.0 \\
87.4\end{array}$ & $\begin{array}{r}80.7 \\
20.5 \\
41.5 \\
59.6 \\
135.3 \\
55.4 \\
79.8\end{array}$ \\
\hline Net iending (m:nus = reimbursement) & 0.0 & 3.0 & 0.7 & .0 .7 & 1.8 & 4.5 & 0.3 & 0.4 \\
\hline Primayy balance (narrow definitoon) $2 ;$ & 18.0 & 23.1 & 44.0 & 39.6 & 64.2 & 59.6 & 45.5 & 23.2 \\
\hline $\begin{array}{l}\text { Interest } \\
\text { internal debt } \\
\text { Ex:ernal debt }\end{array}$ & $\begin{array}{r}26.2 \\
3.7 \\
22.5\end{array}$ & $\begin{array}{r}27.9 \\
2.7 \\
25.2\end{array}$ & $\begin{array}{r}27.4 \\
32 \\
24.1\end{array}$ & $\begin{array}{r}21 .: \\
1.7 \\
18.4\end{array}$ & $\begin{array}{r}14.3 \\
2.3 \\
12.0\end{array}$ & $\begin{array}{r}13.5 \\
1.9 \\
11.6\end{array}$ & $\begin{array}{r}14.1 \\
1.6 \\
12.4\end{array}$ & $\begin{array}{r}15.2 \\
1.8 \\
13.5\end{array}$ \\
\hline Dveral[ balasce (payment orcer basis) & -58.1 & -72.7 & -48.5 & -52.5 & -14.4 & -23.1 & -56.3 & -72.3 \\
\hline $\begin{array}{l}\text { Chunge in arrcars } \\
\text { Externat deb: (psincipal and interest payments) ji } \\
\text { Domestic ‘ebt }\end{array}$ & $\begin{array}{r}-10.6 \\
0.0 \\
.10 .6\end{array}$ & $\begin{array}{r}-16.9 \\
0.0 \\
-16.9\end{array}$ & $\begin{array}{r}-13.7 \\
11.9 \\
-25.6\end{array}$ & $\begin{array}{r}-7.9 \\
7.9 \\
-15.8\end{array}$ & $\begin{array}{l}-41.6 \\
-30.0 \\
-11.6\end{array}$ & $\begin{array}{r}-24.6 \\
-13.3 \\
-11.3\end{array}$ & $\begin{array}{l}-30.6 \\
-14.8 \\
-15.8\end{array}$ & $\begin{array}{r}-6.7 \\
0.0 \\
-6.7\end{array}$ \\
\hline Payments during complementary period'float & 0.0 & 0.0 & .5 .9 & -6.6 & .9 .0 & 3.2 & $-c .6$ & 8.4 \\
\hline Overall baiance (cash basis) & -68.7 & -89.6 & -68.1 & -66.9 & -65.0 & -44.5 & -87.5 & -70.6 \\
\hline Financing & 68.7 & 89.6 & 68.1 & 66.9 & 65.1 & 44.6 & 87.5 & 70.6 \\
\hline $\begin{array}{l}\text { Domestic financing } \\
\text { Banis financing } \\
\text { Nel use of Fund resources } \\
\text { Disbursemen:s } \\
\text { Repayments } \\
\text { Oiher } \\
\text { Nonbänix Inancirg }\end{array}$ & $\begin{array}{r}-13.4 \\
-9.3 \\
13.9 \\
14.4 \\
-0.5 \\
-23.2 \\
-4.1\end{array}$ & $\begin{array}{r}6.0 \\
6.1 \\
5.9 \\
6.9 \\
-1.0 \\
0.2 \\
0.0\end{array}$ & $\begin{array}{r}-i 8.2 \\
-16.7 \\
9.2 \\
10.1 \\
-0.9 \\
-25.9 \\
-1.5\end{array}$ & $\begin{array}{r}3.5 \\
-1.2 \\
1.1 \\
3.6 \\
-2.5 \\
-2.3 \\
4.6\end{array}$ & $\begin{array}{r}.25 .0 \\
-26.3 \\
-2.4 \\
0.0 \\
-2.4 \\
-21.9 \\
0.3\end{array}$ & $\begin{array}{r}-35.8 \\
-46.3 \\
0.6 \\
6.1 \\
-5.5 \\
-46.9 \\
10.5\end{array}$ & $\begin{array}{r}13.9 \\
1.2 \\
-2.5 \\
6.3 \\
-8.9 \\
3.8 \\
12.6\end{array}$ & $\begin{array}{r}-49.0 \\
-47.8 \\
-3.0 \\
7.5 \\
-10.5 \\
-44.7 \\
-1.3\end{array}$ \\
\hline $\begin{array}{l}\text { Exiernal financing } \\
\text { Praject linancing } \\
\text { Grants } \\
\text { Loans } \\
\text { Amortization due } \\
\text { Program aid } \\
\text { Grants } \\
\text { Loarts } \\
\text { Debt relief obtained 4i }\end{array}$ & $\begin{array}{r}82.0 \\
45.8 \\
21.5 \\
24.3 \\
-18.3 \\
30.9 \\
17.2 \\
13.8 \\
23.7\end{array}$ & $\begin{array}{r}83.6 \\
62.0 \\
26.6 \\
35.4 \\
-16.2 \\
15.6 \\
13.8 \\
1.8 \\
22.8\end{array}$ & $\begin{array}{r}86.3 \\
62.1 \\
23.8 \\
38.2 \\
-17.3 \\
33.5 \\
20.6 \\
12.9 \\
8.0\end{array}$ & $\begin{array}{r}63.5 \\
69.7 \\
42.1 \\
27.7 \\
-20.5 \\
14.2 \\
14.2 \\
0.0 \\
0.0\end{array}$ & $\begin{array}{r}91.1 \\
58.0 \\
34.9 \\
23.0 \\
-20.4 \\
17.0 \\
8.3 \\
8.7 \\
36.5\end{array}$ & $\begin{array}{r}80.3 \\
63.0 \\
33.9 \\
29.1 \\
-23.6 \\
27.6 \\
17.2 \\
10.4 \\
13.3\end{array}$ & $\begin{array}{r}73.7 \\
74.4 \\
25.7 \\
49.1 \\
-22.9 \\
1.7 \\
1.7 \\
0.0 \\
20.5\end{array}$ & $\begin{array}{r}119.6 \\
79.8 \\
24.5 \\
55.3 \\
-17.5 \\
41.6 \\
21.5 \\
20.1 \\
15.7\end{array}$ \\
\hline Finarcing gap & 0.0 & 0.0 & 0.0 & 0.0 & 0.0 & 0.0 & 0.0 & 0.0 \\
\hline Memorandum iterns: & \multicolumn{8}{|c|}{ (In porcent of GDP, unjess otherwise indicated) } \\
\hline $\begin{array}{l}\text { Total revenue } \\
\text { Tolal exponditure } \\
\text { Of which }\end{array}$ & $\begin{array}{l}12.8 \\
19.8\end{array}$ & $\begin{array}{l}14.9 \\
22.1\end{array}$ & $\begin{array}{l}15.2 \\
19.5\end{array}$ & $\begin{array}{l}14.6 \\
18.8\end{array}$ & $\begin{array}{l}15.5 \\
16.6\end{array}$ & $\begin{array}{l}16.0 \\
17.6\end{array}$ & $\begin{array}{l}16.6 \\
20.1\end{array}$ & $\begin{array}{l}16.2 \\
20.3\end{array}$ \\
\hline $\begin{array}{l}\text { Wages } \\
\text { Public investment }\end{array}$ & $\begin{array}{l}5.5 \\
6.5\end{array}$ & $\begin{array}{l}5.4 \\
7.8\end{array}$ & $\begin{array}{l}5.2 \\
6.4\end{array}$ & $\begin{array}{l}5.0 \\
6.9\end{array}$ & $\begin{array}{l}4.7 \\
5.9\end{array}$ & $\begin{array}{l}4.5 \\
6.3\end{array}$ & $\begin{array}{l}4.7 \\
7.6\end{array}$ & $\begin{array}{l}4.6 \\
7.8\end{array}$ \\
\hline Overall balanze (payment order basis, exci. grants! & -7.0 & -7.2 & -4.3 & -4.2 & $\cdot 0.1$ & -1.6 & -3.5 & -4.2 \\
\hline $\begin{array}{l}\text { Primary balance (narrow definition) } 2 / \\
\text { Primary balance (uread definition) }\end{array}$ & $\begin{array}{r}2.2 \\
-3.8\end{array}$ & $\begin{array}{r}2.3 \\
-4.5\end{array}$ & $\begin{array}{r}3.9 \\
-9.9\end{array}$ & $\begin{array}{r}3.2 \\
-2.5\end{array}$ & $\begin{array}{l}4.7 \\
0.0\end{array}$ & $\begin{array}{r}4.1 \\
-0.7\end{array}$ & $\begin{array}{r}2.8 \\
-2.6\end{array}$ & $\begin{array}{r}1.3 \\
.3 .3\end{array}$ \\
\hline GDP $5 /$ & 831.1 & $1,002.9$ & $1,129.5$ & $1,249.8$ & $1,360,6$ & 1.469 .9 & $1,605.4$ & 1.738 .6 \\
\hline
\end{tabular}

Sources: Beninese authorities; and staff estimates.

1/ Including value-added taxes an imgorts.

2/ Tolal reverue minus total expendiaure, exciuding investonent finarced from abroad, interest sayments, and net lending.

3/ Arrears on debt service stbject to relief from non-Paris Club creditors.

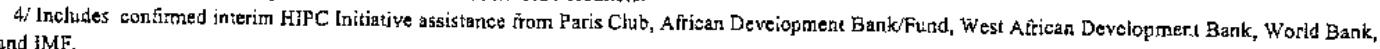
and $\mathrm{MMF}$.

Si In billions of CFA francs. 
Table 17. Benin: Central Government Revenue, 1994-2001

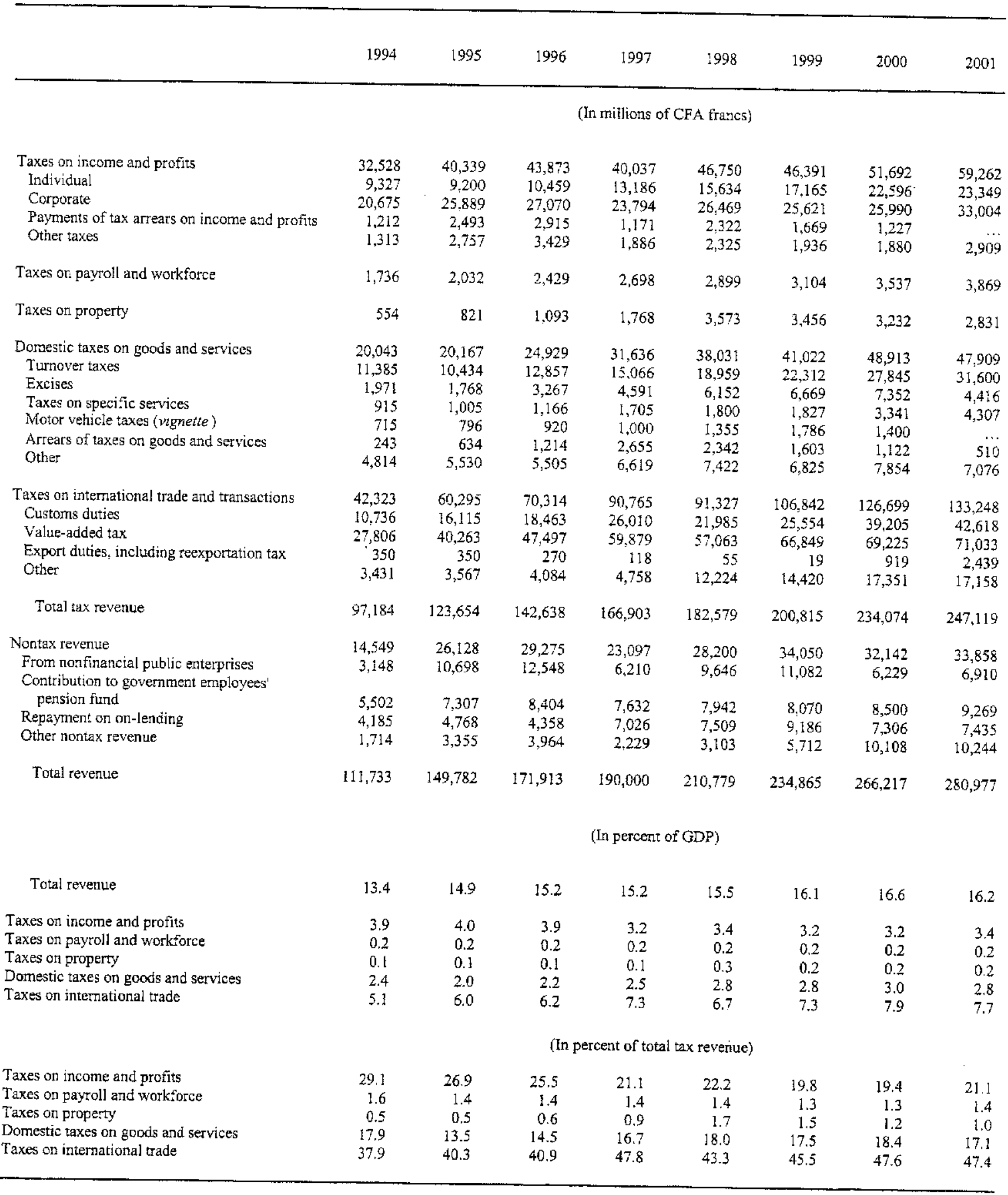

Sources: Berinese authorities; and staff estimates. 
Table 18. Benin: Central Government Expenditure, 1994-2001

\begin{tabular}{|c|c|c|c|c|c|c|c|c|}
\hline . & 1994 & 1995 & 1996 & 1997 & 1998 & 1999 & 2000 & 2001 \\
\hline \multicolumn{9}{|c|}{ (In bilition of CFA francs) } \\
\hline Total expenditure & 164.4 & 221.9 & 220.4 & 234.4 & 225.2 & 258.1 & 322.3 & 353.2 \\
\hline Primary expenditure & 88.3 & 126.0 & 127.9 & 142.3 & 146.6 & 175.4 & 220.6 & 257.7 \\
\hline Salaries, pensions, and scholarships & 56.1 & 65.4 & 75.0 & 78.3 & 81.4 & 84.5 & 94.1 & 101.2 \\
\hline Wage bill & 45.7 & 53.9 & 53.2 & 62.0 & 64.6 & 66.3 & 74.8 & 80.7 \\
\hline Pensions and scholarstips & 10.4 & 11.5 & 16.9 & 16.2 & 16.8 & 18.3 & 19.3 & 20.5 \\
\hline Other expenditure and current transfers & 27.9 & 47.5 & 45.5 & 50.1 & 47.8 & 62.9 & 91.5 & 101.1 \\
\hline 3udgetary contribution to :nvestment & 4.3 & 13.2 & 7.3 & 13.9 & 17.4 & 28.0 & 35.0 & 55.4 \\
\hline Interest & 26.2 & 27.9 & 27.4 & 21.1 & 14.3 & 13.5 & 14.0 & 15.3 \\
\hline Internal debt & 3.7 & 2.7 & 3.2 & 2.7 & 2.3 & 1.9 & 1.6 & 1.8 \\
\hline Externa: debt & 22.5 & 25.2 & 24.1 & 18.4 & 12.0 & 11.6 & 12.6 & 13.5 \\
\hline Investment expenditure financed from abroad & 49.9 & 65.0 & 64.5 & 718 & 62.5 & 64.8 & 87.4 & 79.8 \\
\hline Net lending $(-=$ reimburscment $)$ & 0.0 & 3.0 & 0.7 & -0.7 & 1.8 & 4.5 & 0.3 & 0.4 \\
\hline & \multicolumn{8}{|c|}{ (In percent of GDP) } \\
\hline Total expenditure & 19.7 & 22.1 & 19.5 & 18.8 & 16.6 & 17.7 & 20.1 & 20.3 \\
\hline Primary expenditure & 10.6 & 12.6 & 11.3 & 11.4 & 10.8 & 12.0 & 13.7 & 14.8 \\
\hline Salaries, pensions, and scholarships & 6.8 & 6.5 & 6.6 & 6.3 & 6.0 & 5.8 & 5.9 & 5.8 \\
\hline Wage bill & 5.5 & 5.4 & 5.1 & 5.0 & 4.7 & 4.5 & 4.7 & 4.6 \\
\hline Persions and scholarships & 1.3 & I.1 & {$[.5$} & 1.3 & 1.2 & 1.3 & 1.2 & 1.2 \\
\hline Other expenditure and current transfers & 3.4 & 4.7 & 4.0 & 4.2 & 3.5 & 4.3 & 5.7 & 5.8 \\
\hline Budgetary contrioution to investment & 0.5 & 1.3 & 0.6 & 1.0 & 1,3 & 1.9 & 2.2 & 3.2 \\
\hline Interest & 3.2 & 2.8 & 2.4 & 1.7 & 1.1 & 0.9 & 0.9 & 0.9 \\
\hline [nterna] debt & 0.4 & 0.3 & 0.3 & 0.2 & 0.2 & 0.1 & 0.1 & 0.1 \\
\hline External debt & 2.7 & 2.5 & 2.1 & 1.5 & 0.9 & 0.8 & 0.8 & 0.8 \\
\hline \multirow{3}{*}{$\begin{array}{l}\text { Investment expenditure financed from abroad } \\
\text { Niet lending ( }=\text { =reimbursement) }\end{array}$} & 6.0 & 6.5 & 5.7 & 5.8 & 4.6 & 4.4 & 5.4 & 4.6 \\
\hline & 0.0 & 0.3 & 0.1 & -0.1 & 0.1 & 0.3 & 0.0 & 0.0 \\
\hline & \multicolumn{8}{|c|}{ (In percest of total expenditure) } \\
\hline Total expenditure & 100.0 & 100.0 & 100.0 & 100.0 & 100.0 & 100.0 & 100.0 & 100.0 \\
\hline Primary expenditure & 53.7 & 56,8 & 58.0 & 60.7 & 65.1 & 68.0 & 68.4 & 73.0 \\
\hline Salaries, pensions, and scholarships & 34.1 & 29.5 & 34.1 & 33.4 & 36.1 & 32.8 & 29.2 & 28.7 \\
\hline Wage bill & 27.8 & 24.3 & 26.4 & 26.5 & 28.7 & 25.7 & 23,2 & 22.8 \\
\hline Pensions and scholarships & 6.3 & 5.2 & 7.7 & 6.9 & 7.5 & 7.1 & 6.0 & 5.8 \\
\hline Other expenditure and current transfers & 17.0 & 21.4 & 20.6 & 22.2 & 21.2 & 24.4 & 28.4 & 28.6 \\
\hline Budgetary contribution to investment & 2.6 & 6.0 & 3.3 & 5.1 & 7.7 & 10.8 & 10.9 & 15.7 \\
\hline Incerest & [5.9 & 12.6 & 12.4 & 9.0 & 6.4 & 5.2 & 4.3 & 4.3 \\
\hline Internal debt & 2.3 & 1.2 & 1.5 & 1.1 & 1.0 & 0.7 & 0.5 & 0.5 \\
\hline External debt & 13.7 & 13.4 & 11.0 & 7.8 & 5.3 & 4.5 & 3.8 & 3.8 \\
\hline Investment expenditure financed from abroad & 30.3 & 29.3 & 29.3 & 30.6 & 27.7 & 25.1 & 27.1 & 22.6 \\
\hline Net lending ( $-=$ reimbursement) & 0.0 & 1.3 & 0.3 & -0.3 & 0.8 & 1.7 & 0.1 & 0.1 \\
\hline
\end{tabular}

Sources: Beninese authoritics; and staff estimates. 
Table 19. Benin: Centra] Government Investment Expenditure, 1994-2001

(In billions of CFA francs)

\begin{tabular}{|c|c|c|c|c|c|c|c|c|}
\hline & 1994 & 1995 & 1996 & 1997 & 1998 & 1999 & 2000 & 2001 \\
\hline Expenditure & 54.9 & 78.3 & 71.8 & 83.7 & 77.4 & 87.1 & 117.5 & 121.0 \\
\hline Rural development & 10.1 & 16.1 & 12.9 & 16.1 & 16.6 & 12.5 & 16.4 & 9.2 \\
\hline Industry & 1.3 & 1.4 & 2.0 & 2.0 & 2.0 & 0.8 & 0.9 & 6.0 \\
\hline Infrastructure & 19.6 & 38.4 & 38.7 & 40.4 & 33.3 & 31.4 & 48.0 & 53.2 \\
\hline Tourism and commerce & 0.9 & 5.1 & 0.3 & 0.9 & 1.7 & 1.6 & 3.5 & 2.6 \\
\hline Health & 7.4 & 7.1 & 6.8 & 5.3 & 6.8 & 12.3 & 15.0 & 17.0 \\
\hline Education & 2.4 & 1.5 & 4.8 & 6.3 & 5.7 & 6.7 & 10.3 & 15.8 \\
\hline Other & 13.2 & 8.7 & 6.3 & 12.8 & 11.4 & 21.8 & 23.4 & 17.2 \\
\hline Financing & 54.9 & 78.3 & 71.8 & 83.7 & 77.4 & 87.1 & 117.5 & 121.0 \\
\hline Domestic financing & 9.1 & 16.3 & 9.8 & 13.9 & 16.4 & 22.3 & 30.0 & 41.7 \\
\hline National budget & 3.7 & 13.2 & 7.1 & 11.7 & 15.0 & 20.0 & 29.1 & 41.3 \\
\hline CAAFNI I/ & 5.4 & 0.0 & 0.0 & 0.0 & 0.0 & 0.0 & 0.0 & 0.0 \\
\hline Other & 0.0 & 3.1 & 2.7 & 2.2 & 1.4 & 2.3 & 0.9 & 0.3 \\
\hline External financing & 45.8 & 62.0 & 62.0 & 69.8 & 61.0 & 64.8 & 87.5 & 79.3 \\
\hline Grants & 21.5 & 26.6 & 23.8 & 42.1 & 36.4 & 30.0 & 30.9 & 24.5 \\
\hline Loans & 24.3 & 35.4 & 38.2 & 27.7 & 24.5 & 34.8 & 56.5 & 54.8 \\
\hline
\end{tabular}

Sources: Ministry of Planning and Statistics, Directorate for Planning and Technical Assistance; and staff estimates.

1/ $\mathrm{CAA}=$ Autonomous Arnortization Fund; FNI $=$ National Investment Fund. 
Table 20. Benin: Operations of the Social Security Fund, 1994-2001

(In millions of CFA francs)

\begin{tabular}{|c|c|c|c|c|c|c|c|c|}
\hline & 1994 & 1995 & 1996 & 1997 & 1998 & 1999 & 2000 & 2001 \\
\hline Contributions & 5,408 & 6,708 & 8,098 & 9,720 & 11,126 & 11,184 & 12,965 & 15,557 \\
\hline Family allowances & 2,271 & 2,817 & 3,401 & 4,082 & 4,673 & 5,000 & 5,509 & 6,534 \\
\hline Workers' compensation & 595 & 738 & 891 & 1,069 & 1,224 & 1,174 & 1,304 & 1,711 \\
\hline Retirement & 2,542 & 3,153 & 3,806 & 4,569 & 5,229 & 5,010 & 6,152 & 7,312 \\
\hline Other revenue & 129 & 360 & 592 & 1,985 & 1,801 & 1,684 & 10,100 & 2,235 \\
\hline Total receipts & 5,537 & 7,068 & 8,690 & 11,705 & 12,927 & 13,516 & 23,065 & 17,792 \\
\hline Benefits & 2,887 & 3,093 & 3,699 & 3,903 & 4,372 & 5,520 & 5,220 & 5,768 \\
\hline Family allowances & 537 & 527 & 863 & 783 & 937 & 1,304 & 882 & 864 \\
\hline Workers' compensation & 114 & 126 & 121 & 119 & 160 & 200 & 169 & 188 \\
\hline Retirement & 2,236 & $2,4 \triangle 0$ & 2,715 & 3,001 & 3,275 & 4,015 & 4,169 & 4,716 \\
\hline Other expenses & 1,031 & 1,374 & 1,683 & 1,547 & 3,087 & 1,957 & 8,819 & 2,358 \\
\hline Total expenditure & 3,918 & 4,467 & 5,382 & 5,450 & 7,459 & 7,477 & 14,039 & 8,126 \\
\hline Surplus/deficit (-) & 1,619 & 2,601 & 3,308 & 6,255 & 5,468 & 6,039 & 9,026 & 9,666 \\
\hline
\end{tabular}

Source: Social Security Fund (OBSS). 
Table 21. Benin: Monetary Survey, 1994-2001

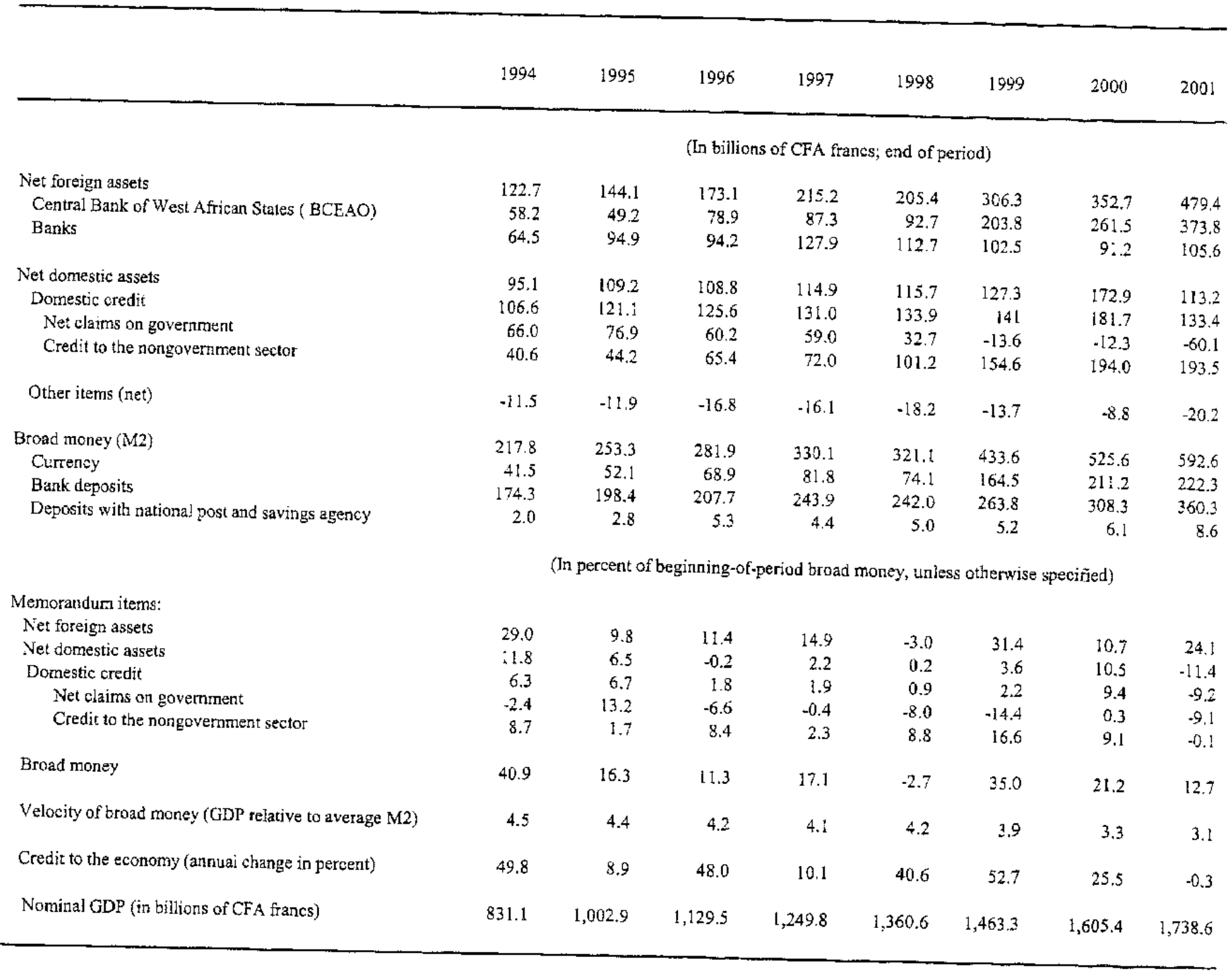

Sources: Central Bank of West African States (BCEAO); and staff estimates. 
Table 22. Benin: Summary Account of the Central Bank (BCEAO), 1994-2001

(En milliards de francs CFA, fin de période)

\begin{tabular}{|c|c|c|c|c|c|c|c|c|}
\hline & 1994 & 1995 & 1996 & 1997 & 1998 & 1999 & 2000 & 2001 \\
\hline Gross foreign assets & 102.1 & 97.1 & 137.1 & 151.5 & 147.1 & 260.8 & 323.5 & 430.3 \\
\hline Gross claims on central government & 40.5 & 53.7 & 53.8 & 55.1 & 52.0 & 52.7 & 52.8 & 51.1 \\
\hline Advances to the treasury & 1.7 & 8.9 & 0.0 & 0.0 & 0.0 & 0.0 & 0.0 & 0.0 \\
\hline Treasury bonds & 0.0 & 0.0 & 0.0 & 0.0 & 0.0 & 0.0 & 1.3 & 1.1 \\
\hline \multicolumn{9}{|c|}{$\begin{array}{l}\text { Trust Funds on-lent and Structural Adjustment } \\
\text { Facility (SAF)/Enhanced Structura! }\end{array}$} \\
\hline Adjustment Facility (ESAF) loans & 38.8 & 44.8 & 53.8 & 55.1 & 52.0 & 52.7 & 51.5 & 50.0 \\
\hline Consolidated credit $1 /$ & 0.0 & 0.0 & 0.0 & 0.0 & 0.0 & 0.0 & 0.0 & 0.0 \\
\hline Other assets & 2.0 & 7.8 & 1.3 & 6.1 & 2.4 & 3.7 & 9.9 & 7.6 \\
\hline Total assets & 144.6 & 160.6 & 194.2 & 212.7 & 201.5 & 317.2 & 386.2 & 489.0 \\
\hline Banknotes and coins outside banks & 41.5 & 52.1 & 68.9 & 81.8 & 74.1 & 164.5 & 211.2 & 222.3 \\
\hline Government deposits and cash & 18.6 & 24.0 & 36.6 & 26.1 & 32.4 & 70.1 & 63.6 & 111.3 \\
\hline Deposits & 17.7 & 23.1 & 35.1 & 25.3 & 31.5 & 68.4 & 60.2 & 109.2 \\
\hline Central government & 10.3 & 10.3 & 34.1 & 25.2 & 31.3 & 68.1 & 59.8 & 105.7 \\
\hline Other public agencies & 7.4 & 12.8 & 2.5 & 0.1 & 0.2 & 0.3 & 0.4 & 3.5 \\
\hline Cash beld by the treasury & 0.7 & 0.9 & 1.5 & 0.8 & 0.9 & 1.7 & 3.4 & 2.1 \\
\hline Commercial banks' deposits and cash & 30.9 & 28.4 & 17.1 & 31.9 & 31.5 & 16.5 & 37.1 & 77.1 \\
\hline Deposits & 26.8 & 24.7 & 10.9 & 24.3 & 25.1 & 7.8 & 25.9 & 63.6 \\
\hline Cash & 4.1 & 3.7 & 6.2 & 7.6 & 6.4 & 8.7 & 11.2 & 13.5 \\
\hline Public enterprises' deposits & 0.5 & 0.4 & 0.5 & 0.5 & 0.5 & 0.6 & 1.1 & 2.6 \\
\hline Short-tern foreign liabilities & 4.4 & 3.1 & 4.4 & 9.1 & 2.4 & 0.8 & 0.7 & 0.7 \\
\hline SDR allocations & 7.6 & 7.3 & 7.0 & 7.5 & 7.6 & 7.9 & 9.0 & 8.8 \\
\hline Long-term foreign liabilities $2 /$ & 39.5 & 44.8 & 53.8 & 55.1 & 52.0 & 56.2 & 61.3 & 57.2 \\
\hline Other liabilities & 1.6 & 0.5 & 5.9 & 0.7 & 1.0 & 0.6 & 2.2 & 9.0 \\
\hline Total liabilities & 144.6 & 160.6 & 194.2 & 212.7 & 201.5 & 317.2 & 386.2 & 489.0 \\
\hline
\end{tabular}

Source: Central Bank of West African States (BCEAO).

1/ The BCEAO rescheduled these claims in 1993 and issued bonds that are held by banks.

2/ Mainly Trust Fund and outstanding SAF/ESAF loans. 
Table 23. Benin: Summary Account of Commercial Banks, 1994-2001

(In billions of CFA francs; end of period)

\begin{tabular}{|c|c|c|c|c|c|c|c|c|}
\hline & 1994 & 1995 & 1996 & 1997 & 1998 & 1999 & 2000 & 2001 \\
\hline Reserves of commercial banks & 30.1 & 28.4 & 17.1 & 31.9 & 31.5 & 16.5 & 37.1 & 77.1 \\
\hline Cash in vault & $\cdots$ & 3.7 & 6.2 & 7.6 & 6.4 & 8.7 & 11.2 & 13.5 \\
\hline Deposits at Central Bank of West & & & & & & & & \\
\hline African States (BCEAO) & $\cdots$ & 24.7 & 10.9 & 24.3 & 25.1 & 7.8 & 25.9 & 63.6 \\
\hline Gross foreign assets & 76.3 & 117.1 & 142.6 & 156.5 & $: 62.4$ & 170.6 & 151.0 & 178.5 \\
\hline Gross claims on government & 36.0 & 34.6 & 36.9 & 32.9 & 30.7 & 25.9 & 25.8 & 23.0 \\
\hline Central administration & 36.0 & 34.5 & 36.0 & 32.6 & 29.6 & 25.9 & 25.8 & 22.3 \\
\hline Other public agencies & 0.0 & 0.1 & 0.9 & 0.3 & 1.1 & 0.0 & 0.0 & 0.7 \\
\hline Credit to the nongovernment sector & 40.6 & 44.2 & 65.4 & 72.0 & 101.2 & 154.6 & 194.0 & 192.8 \\
\hline Petforming credit & $\cdots$ & 40.4 & 62.9 & 66.4 & 97.9 & 145.6 & 183.6 & 184.5 \\
\hline Crop eredit & $\cdots$ & 0.0 & 5.5 & 0.0 & 0.0 & 2.3 & 0.5 & 2.4 \\
\hline Other & $\cdots$ & 40.4 & 57.4 & 66.4 & 97.9 & 143.3 & 118.6 & 182.1 \\
\hline Nonperforming credit & $\cdots$ & 3.8 & 2.5 & 5.6 & 3.3 & 9.0 & 10.5 & 8.3 \\
\hline Other assets & 7.8 & 26.4 & 30.6 & 20.6 & 22.3 & 31.3 & 27.4 & 17.5 \\
\hline Total assets & 190.8 & 250.6 & 291.7 & 313.9 & 348.1 & 398.9 & 435.4 & 488.9 \\
\hline Private sector deposits & 137.0 & 159.7 & 178.1 & 207.7 & 207.0 & 216.1 & 261.6 & 304.1 \\
\hline Short-term foreign liabilities & 11.8 & 22.2 & 48.4 & 28.6 & 49.7 & 68.1 & 59.8 & 72.9 \\
\hline Banks & 2.2 & 3.8 & 7.0 & 5.1 & 7.9 & 24.8 & 9.4 & 18.4 \\
\hline Cal1 accounts & 8.2 & 9.1 & 24.9 & 13.9 & 17.4 & 15.5 & 18.9 & $\$ 7.9$ \\
\hline Other & 1.4 & 9.3 & 14.5 & 9.6 & 25.5 & 27.8 & 31.5 & 36.6 \\
\hline Government deposits & 30.7 & 28.4 & 27.4 & 42.7 & 56.0 & 74.4 & 79.4 & 84,4 \\
\hline Central administration & 1.3 & 2.9 & 0.8 & 7.1 & $2 ! .7$ & 27.5 & 33.8 & 34.3 \\
\hline Other public agencies & 29.4 & 25.5 & 26.6 & 35.6 & 34.3 & 46.8 & 45.6 & 50.1 \\
\hline Central bank refinancing & 0.0 & 0.0 & 2.0 & 1.0 & 0.0 & 0.0 & 0.0 & 0.0 \\
\hline Long-term foreign liabilities & 1.3 & 0.5 & 0.6 & 0.8 & 0.7 & 2.4 & 2.3 & 0.9 \\
\hline Revaluation account & 10.0 & 10.0 & 0.0 & 0.0 & 0.0 & 0.0 & 0.0 & 0.0 \\
\hline Dther liabilities & 0.0 & 29.8 & 35.2 & 32.8 & 33.6 & 37.8 & 32.3 & 26.6 \\
\hline rotal liabilities & 190.8 & 250.6 & 291.7 & 313.6 & 348.1 & 398.9 & 435.3 & 488.9 \\
\hline
\end{tabular}


Table 24. Berin: Net Claims on the Centrai Government, 1994-2001 1/

(In billions of CFA francs; end of period)

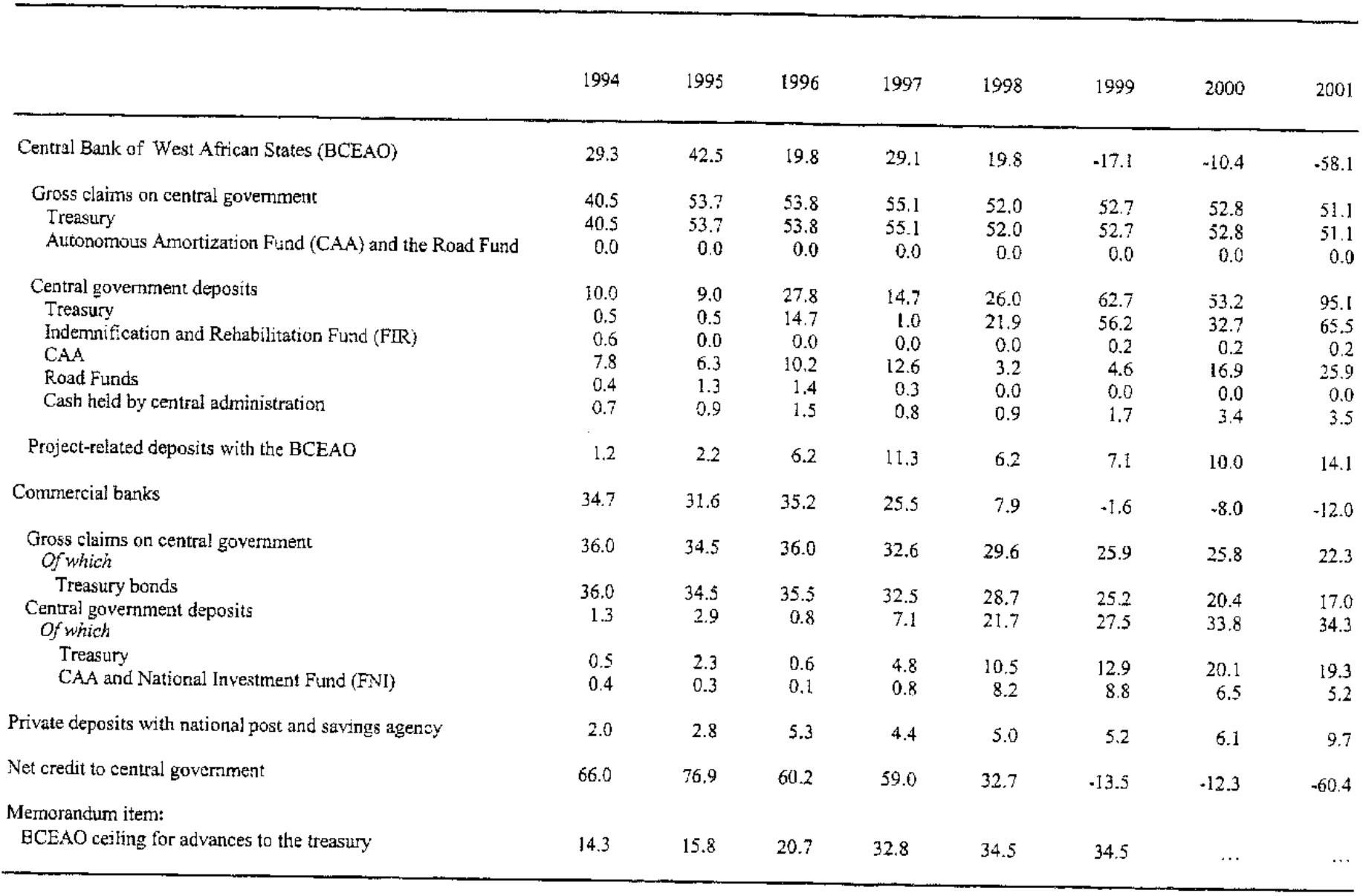

Source: Central Bank of West African States (BCEAO).

b The government includes the treasury, the Autonomous Amortization Fund (CAA), the National investment Fund (FND), and the Road Fund. 
Table 25. Benin: Distribution of Credit to the Economy, 1994-2001 1/

(In millions of CFA francs; end of period)

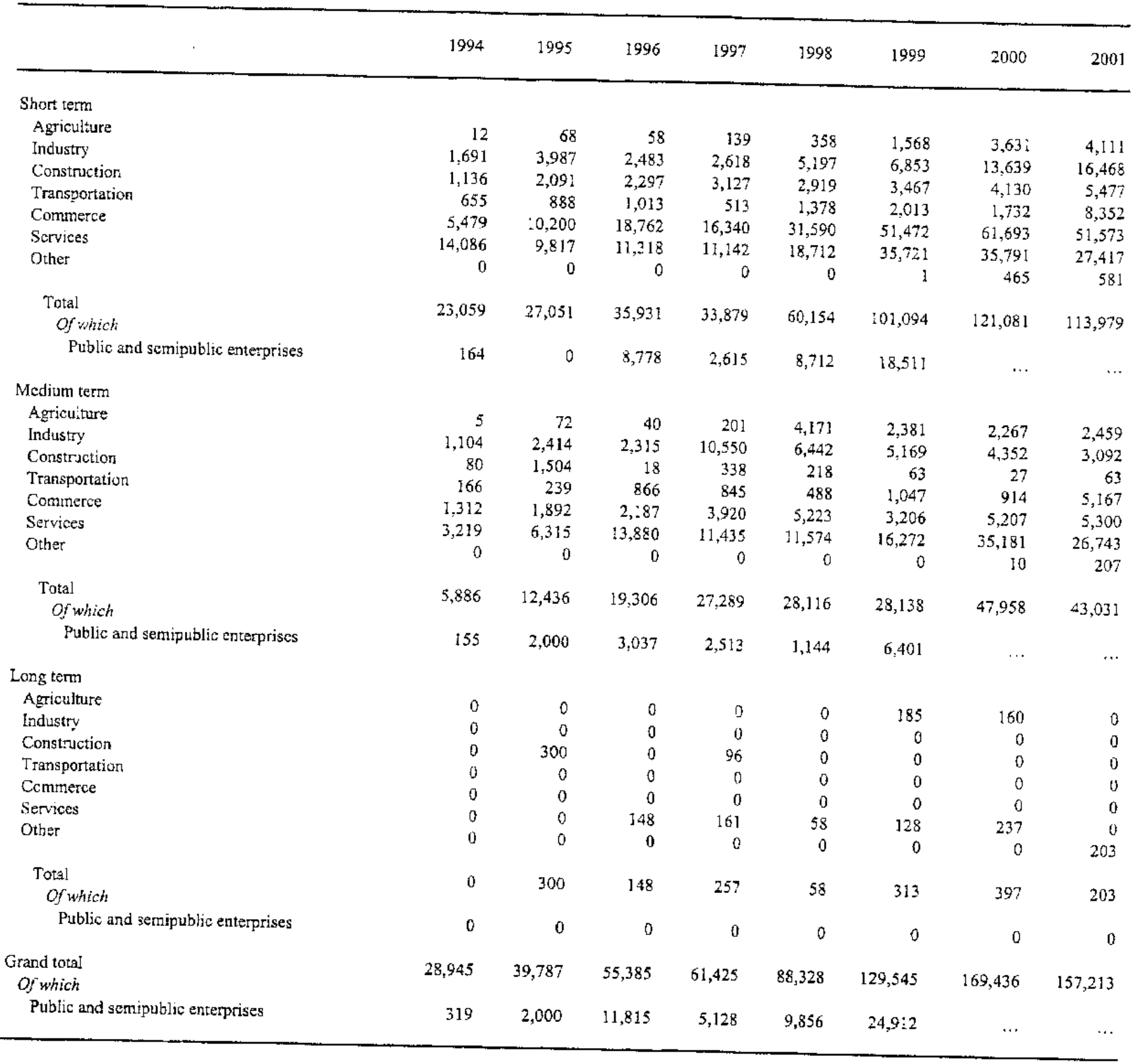

Source: Central Bank of West African States (BCEAO).

1/ New banks, as reported to the Credit Risk Registry (Centrale des Risques), excluding credit of liquidated banks. 
Table 26. Benin: Central Bank Lending Rates, January 1998-December 2001

(Percent per annum; end of period, wniess otherwise indicated)

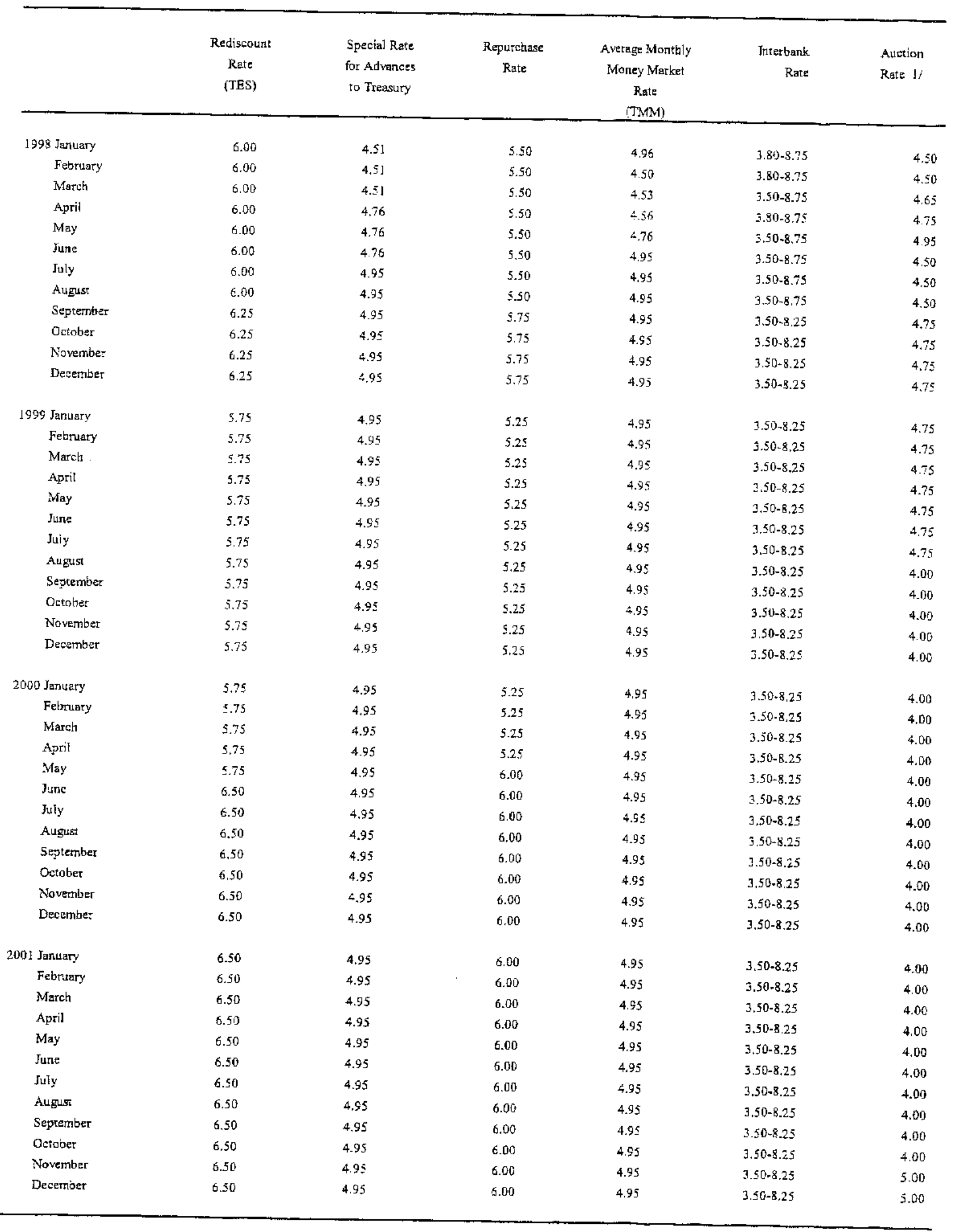

Source: Centrai Bank of West African States (BCEAO).

I/ A weekly auction system on the rnoney market was introduced on October \8, 1993. 
'Tablc 27. Benin: Cormunercial Banks' Interest Rates, December 1998-Decemkter 2001

(In percent per anoum; erd of pertind)

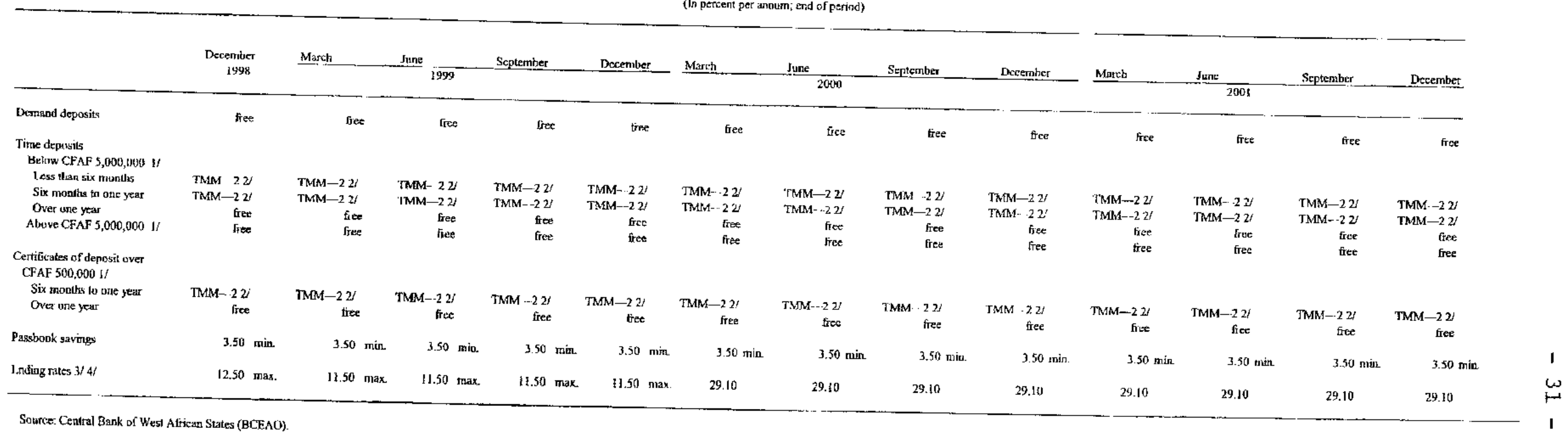

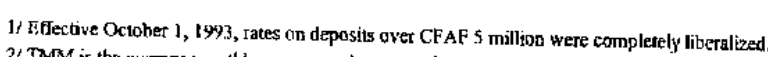

2) TMM is the average inunltily moncy 1 itarket rate published by the BCEAO.

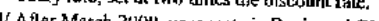

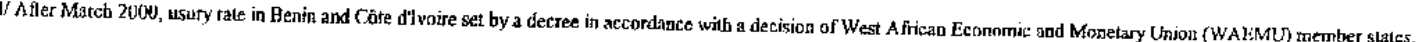


Table 28. Benin: Balance of Payments, 1994-2001

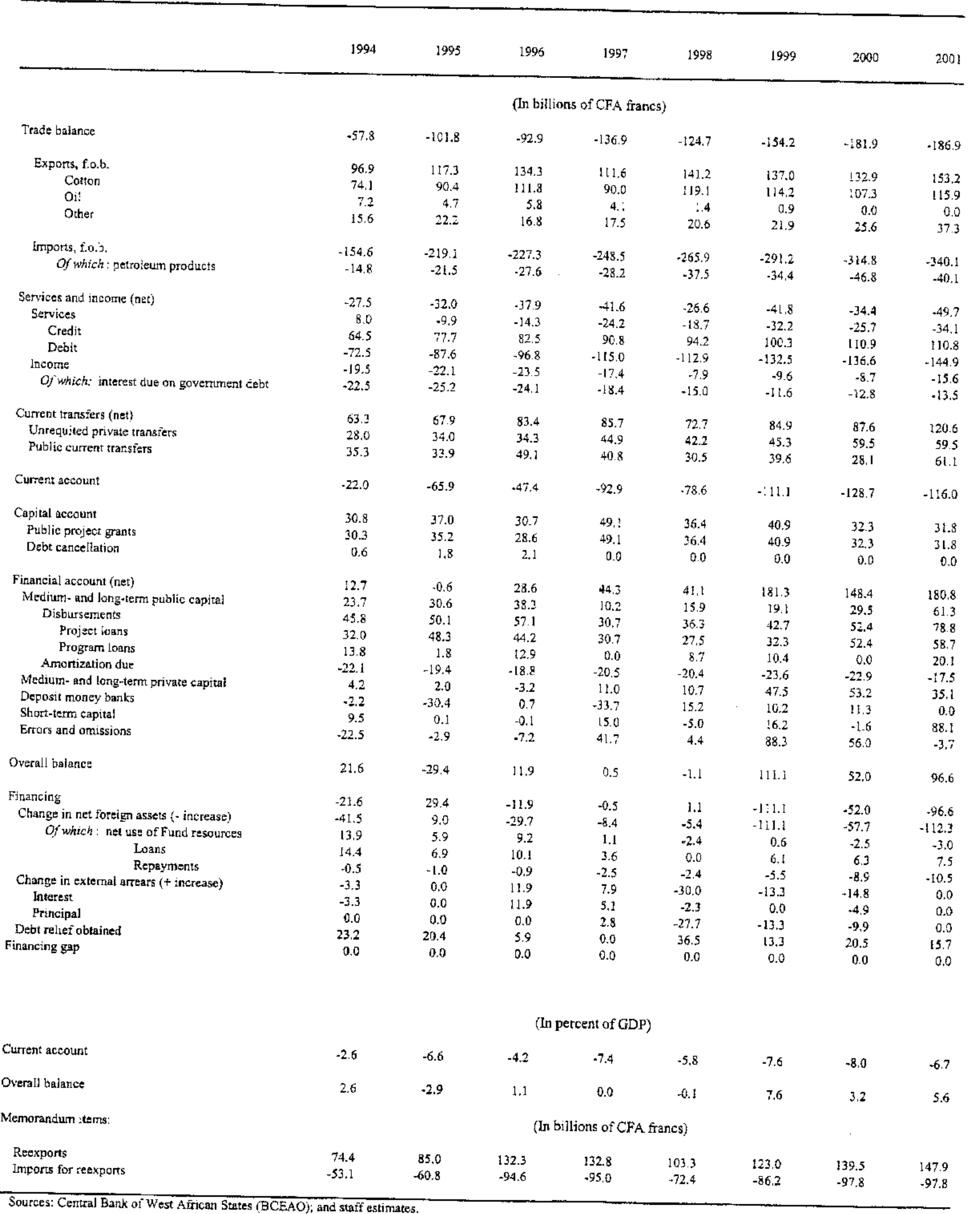


Table 29. Benin: Balance of Payments, 1994-2001

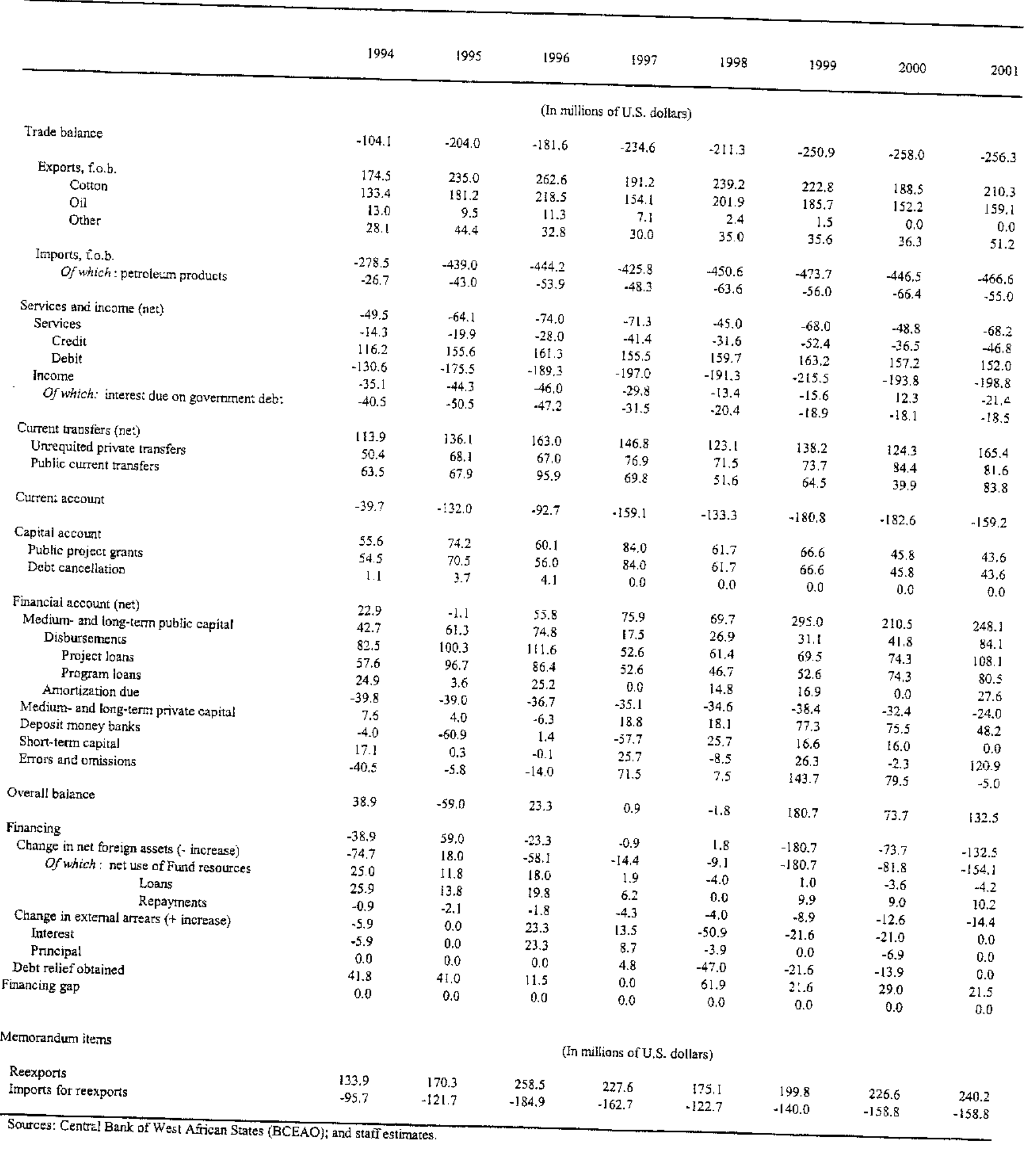


Table 30. Benin: External Trade Indices, 1994-2001

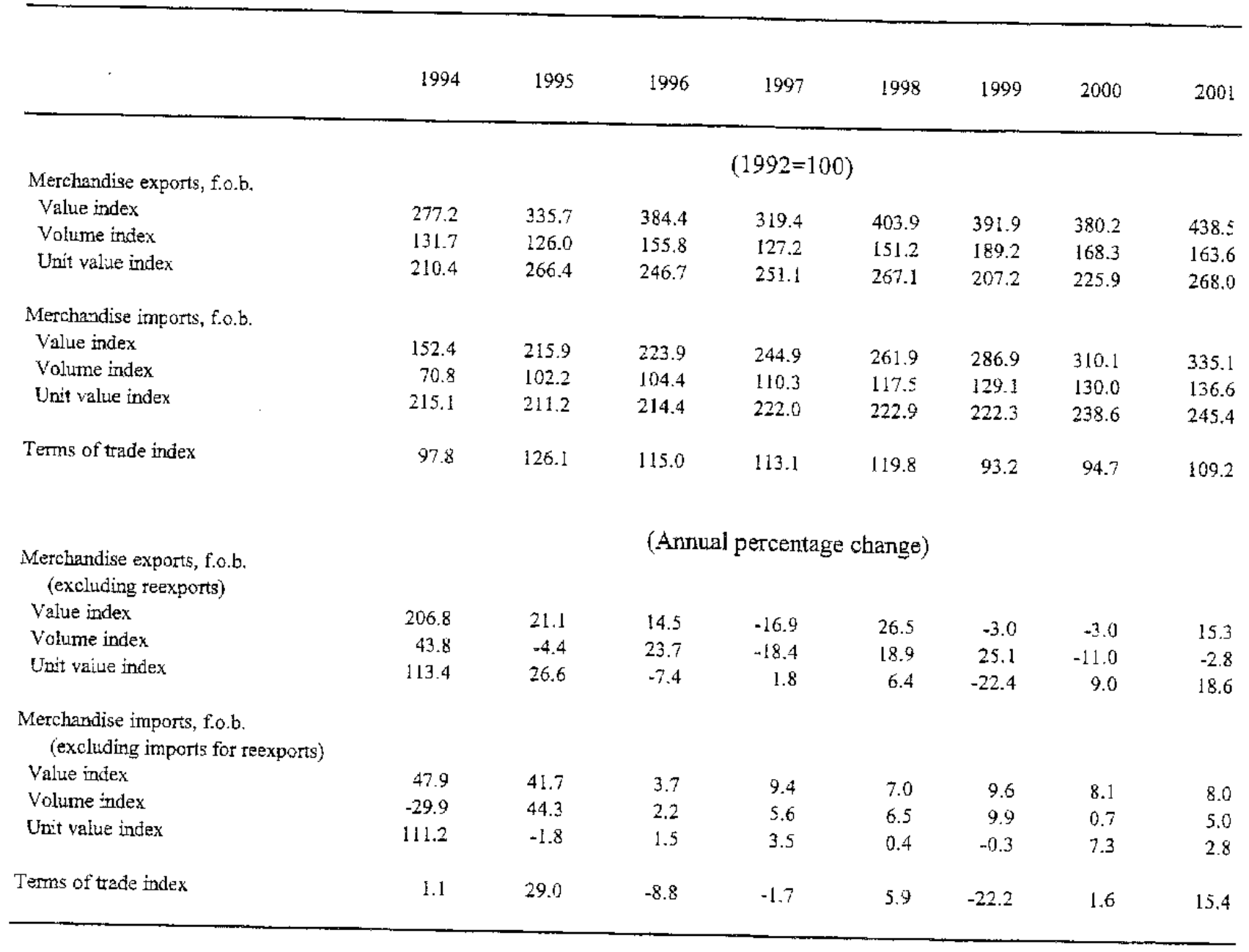

Sources: Beninese authorities; and staff estimates. 
Table 31. Benin: Services, Income, and Private Transfers, 1994-2001

(In billions of CFA francs)

\begin{tabular}{|c|c|c|c|c|c|c|c|c|}
\hline & 1994 & 1995 & 1996 & 1997 & $i 998$ & 1999 & 2000 & 2001 \\
\hline Services (net) & -7.9 & -9.9 & .14 .3 & -24.2 & -18.7 & -32.2 & -25.7 & -34.1 \\
\hline Credit & 64.5 & 77.7 & 82.5 & 90.8 & 94.2 & 100.3 & 110.9 & $: 10.8$ \\
\hline Freight and insurance & 14.3 & 23.1 & 28.8 & $3: 9$ & 19.3 & 33.2 & 18.8 & 36.7 \\
\hline Government operations & 9.9 & 9.5 & 9.7 & 10.5 & 9.0 & 13.2 & 6.9 & 15.7 \\
\hline Tourism & 13.0 & 15.3 & 14.0 & 14.7 & 37.9 & 15.9 & 54.7 & 18.6 \\
\hline Other services & 1.2 & 2.2 & 2.3 & 3.5 & 21.4 & 5.8 & 18.8 & 7.0 \\
\hline Other transport & 26.1 & 27.6 & 27.7 & 30.2 & 6.6 & 32.2 & 11.7 & 32.8 \\
\hline Debit & .72 .5 & -87.6 & -96.8 & -115.0 & -112.9 & -132.5 & -136.6 & -144.9 \\
\hline Freight and insurance & -36.7 & -49.4 & -56.6 & -73.4 & -58.8 & $.64,4$ & -66.8 & -93.5 \\
\hline Government operations & -9.5 & -10.0 & -10.3 & -10.4 & 0.0 & -1.1 & -3.8 & -13.3 \\
\hline Tourism & -3.3 & -4.5 & -4.5 & -4.6 & .11 .9 & -15.8 & -8.7 & -5.8 \\
\hline Other services & +12.7 & -13.3 & -14.5 & -15.0 & -34.5 & -27.2 & -50.5 & .18 .9 \\
\hline Other transport & -10.3 & -10.4 & -10.9 & -11.6 & -7.7 & -24.0 & -6.8 & -13.4 \\
\hline Income & -19.5 & -22.1 & -23.5 & -17.4 & -7.9 & -9.6 & -8.7 & -15.6 \\
\hline Credit & 3.0 & 3.1 & 3.6 & 4.0 & 18.3 & 14.5 & 22.2 & 5.5 \\
\hline $\begin{array}{l}\text { Debit } \\
\text { Of which }\end{array}$ & -22.5 & -25.2 & -27.1 & -21.4 & -26.2 & -24.1 & -30.9 & -21.1 \\
\hline Interest on government debt & -22.5 & -25.2 & -24.1 & -18.4 & -12.0 & $-11,6$ & -12.8 & -13.5 \\
\hline Private transfers & 28.0 & 34.0 & 34.3 & 44.9 & 42.2 & 45.3 & 59.5 & 59.5 \\
\hline Credit & 32.3 & 38.2 & 39.5 & 50.1 & 60.2 & 53.6 & 65.0 & 65.0 \\
\hline Debit & -4.3 & -4.2 & -5.2 & -5.2 & -18.0 & -8.3 & -5.5 & -5.5 \\
\hline
\end{tabular}

Sources: Central Bank of West African States (BCEAO); and staff estimates. 


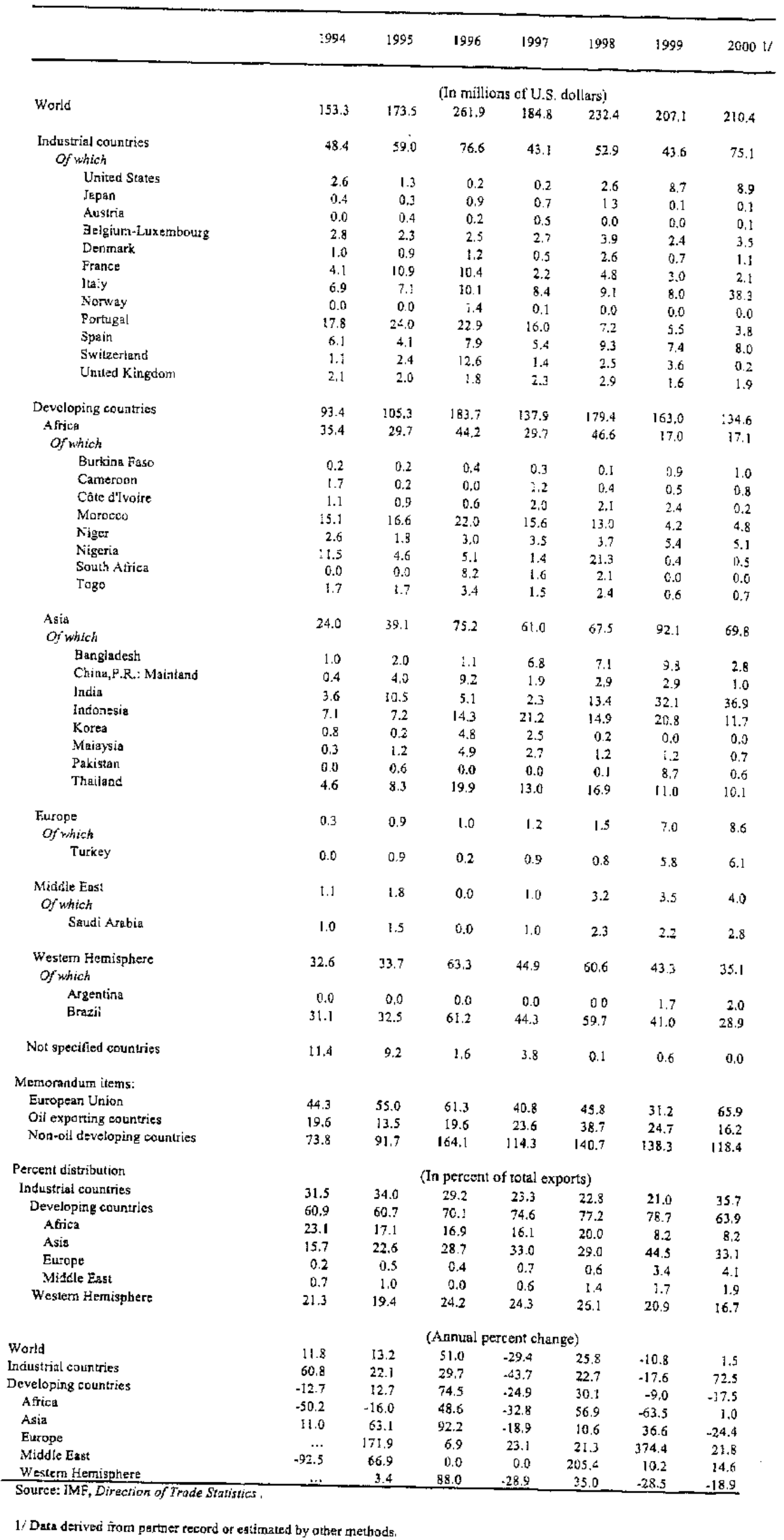


Table 33. Benin: Origin of Recorded Imports, 1994-2000 1/

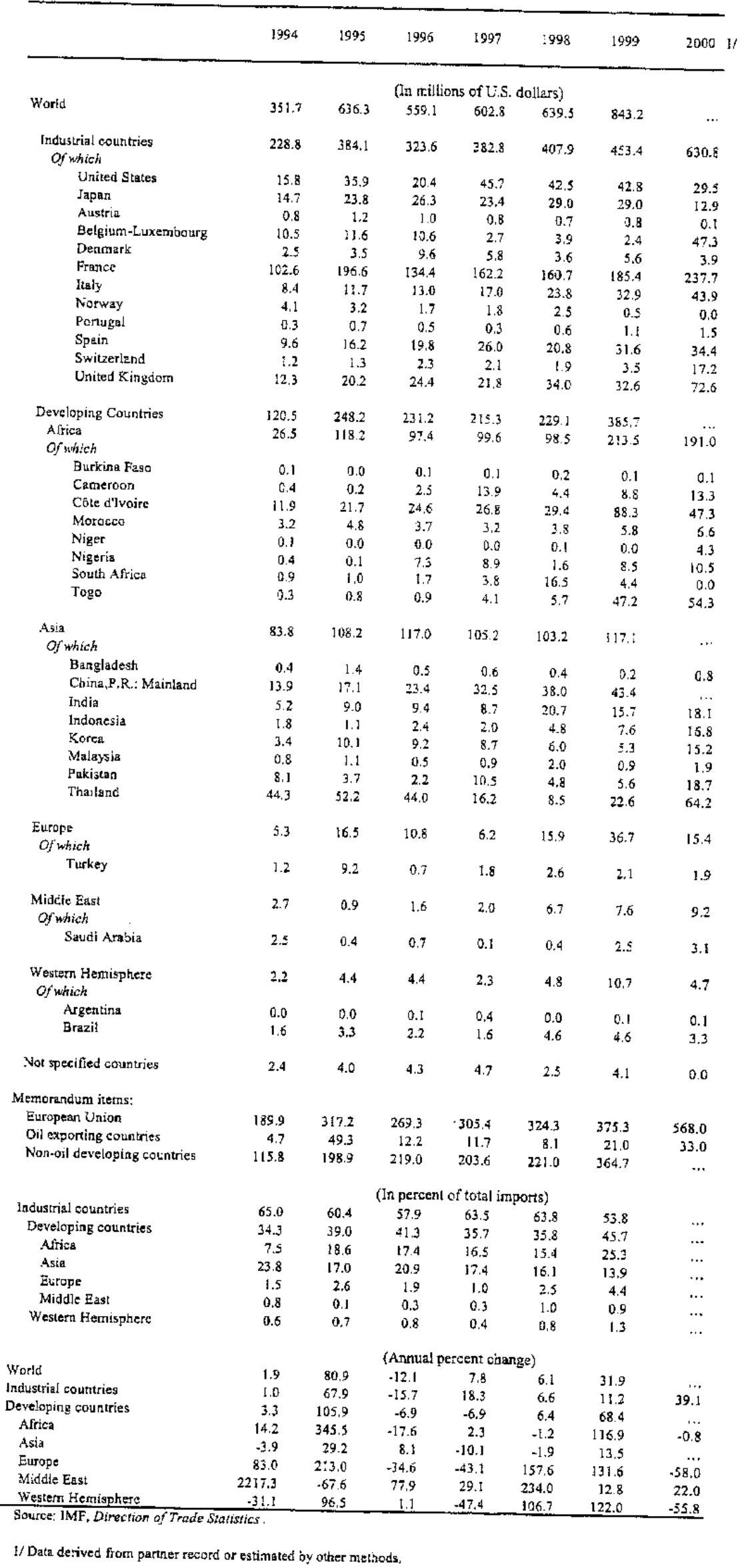


Table 34. Benin: Stock of Pubiic and Guaranteed Extemal Debt, 1994-2001

(In millions of CFA franes)

\begin{tabular}{|c|c|c|c|c|c|c|c|c|}
\hline & 1994 & 1995 & 1996 & 1997 & 1998 & 1999 & 2000 & 2000 \\
\hline Multilateral institutions & 462.2 & 497.0 & 531.0 & 577.5 & 627.2 & 656.5 & 748.7 & 829.0 \\
\hline $\mathrm{IMF}$ & 32.0 & 42.5 & 52.1 & 55.1 & 53.2 & 56.7 & 59.2 & 57.1 \\
\hline IDA & 255.4 & 249.4 & 272.3 & 298.4 & 324.6 & 359.0 & 409.1 & 449.4 \\
\hline African Developrnent Bank/Fuad & 101.7 & 101.5 & 126.6 & $: 25.7$ & 156.8 & 151.4 & 181.4 & 205.2 \\
\hline Other & 73.2 & 103.7 & 80.0 & 98.3 & 92.6 & 89.4 & 99.0 & 117.3 \\
\hline Bilateral creditors & 179.0 & 325.4 & 261.7 & 225.0 & 157.0 & 160.6 & 188.0 & 159.9 \\
\hline Paris Ciub l/ & 164.1 & 221.4 & 116.5 & 115.5 & 86.2 & 81.1 & 131.3 & 136.0 \\
\hline France $2 f$ & 64.0 & 118.6 & 40.2 & 39.4 & 35.7 & 30.2 & 41.9 & $44 . C$ \\
\hline Germany & 2.3 & 2.3 & 1.2 & 1.7 & 1.7 & 1.7 & 1.5 & 1.7 \\
\hline Ilaiy & $\ldots$ & $\ldots$ & 16.4 & 16.6 & 16.6 & 16.6 & 20.6 & 22.3 \\
\hline Norway & 25.2 & 23.2 & 16.6 & 16.1 & 18.1 & 18.3 & 20.0 & 21.0 \\
\hline Russia & 21.0 & 21.0 & 30.2 & 29.1 & 3.6 & 3.6 & 13.0 & 13.8 \\
\hline United Kingdom & 3.0 & 2.9 & 2.4 & 2.6 & 2.6 & 2.7 & 2.8 & 2.9 \\
\hline Other & 48.6 & 53.5 & 9.5 & 10.0 & 7.8 & 8.0 & 31.5 & 30.3 \\
\hline Other creditors $3 /$ & 14.9 & 104.0 & 145.2 & 109.5 & 70.8 & 79.5 & 56.7 & 23.9 \\
\hline Total debt outstanding (before debt relief) & 641.2 & 822.4 & 792.7 & 802.5 & 784.2 & 817.1 & 936.7 & 988.9 \\
\hline \multicolumn{9}{|l|}{ Memorandum item: } \\
\hline Debt ourstanding (in percent of GDP) & 77.2 & 82.0 & 70.2 & 64.2 & 57.6 & 55.6 & 58.3 & 56.9 \\
\hline
\end{tabular}

Solrces: Autonomous Amortization Fund (CAA); and staff estimates.

1/ In 1997, Russiz became a member os the Paris Club.

2/ Including hospital and postal debt.

3 / Excludes dett to Nigeria of which the amount is contested. 
Table 35. Benin: Debt-Service Obligations on Public and Publicly Guaranteed Debt, 1994-2001

(In billions of CFA francs)

\begin{tabular}{|c|c|c|c|c|c|c|c|c|}
\hline & 1994 & 1995 & 1996 & 1997 & 1998 & 1999 & 2000 & 2001 \\
\hline \multicolumn{6}{|l|}{ Interest } & & & \\
\hline Multilaterals (excluding IMF) & 4.6 & 4.8 & 5.1 & $\begin{array}{r}18.4 \\
5.9\end{array}$ & $\begin{array}{r}15.2 \\
5.6\end{array}$ & 11.6 & 12.7 & 13.5 \\
\hline IMF & 0.5 & 0.5 & 0.4 & $\begin{array}{l}5.9 \\
0.6\end{array}$ & 5.6 & 6.0 & 6.6 & 7.3 \\
\hline Paris Club (mediurn-tern debt) & 8.8 & 10.7 & 11.3 & $\begin{array}{l}0.6 \\
4.7\end{array}$ & $\begin{array}{l}0.3 \\
4.9\end{array}$ & 0.2 & 0.7 & 0.3 \\
\hline Other bilateral creditors & 6.8 & 7.5 & 5.8 & $\begin{array}{l}4.7 \\
6.0\end{array}$ & 4.9 & 4.0 & 4.7 & 5.3 \\
\hline Short-te:m debt & 0.0 & 0.0 & 0.0 & $\begin{array}{l}6.0 \\
0.0\end{array}$ & 3.5 & 0,3 & 0.4 & 0.6 \\
\hline Postal and hospital debr & 1.8 & 1.6 & 14 & 0.0 & 0.0 & 0.3 & 0.0 & 0.0 \\
\hline Other & 01 & 01 & & 1.2 & 0.9 & 0.8 & 0.3 & 0.0 \\
\hline & 0.1 & 0.1 & 0.1 & 0.0 & 0.0 & 0.0 & 0.0 & 0.0 \\
\hline \multicolumn{6}{|l|}{ Amortization } & & & \\
\hline Multilaterals (exchuding IMF) & 7.0 & 7.7 & 8.1 & $\begin{array}{r}23.0 \\
9.8\end{array}$ & $\begin{array}{l}22.8 \\
10.8\end{array}$ & 28.8 & 31.7 & 28.0 \\
\hline $\mathrm{MF}$ & 0.5 & 1.0 & 0.9 & & $\begin{array}{r}10.8 \\
2.4\end{array}$ & 12.1 & 14.4 & 15.7 \\
\hline Paris Club (medium-term debt) & 0.7 & 1.2 & 0.9 & 2.5 & 2.4 & 5.2 & 8.9 & 10.5 \\
\hline Other bilateral creditors & 5.6 & 6.0 & 6.1 & 0.1 & 3.0 & 1.0 & 20 & 0.9 \\
\hline Shar-term deb: & 6.1 & 2.0 & 1.0 & 7.7 & 3.3 & 5.3 & 2.6 & 0.9 \\
\hline Postal and hospital deb: & 2.1 & 23 & 1.0 & 0.0 & 0.0 & 0.0 & 0.0 & 0.0 \\
\hline Other & 0.2 & 0.2 & 2.5 & 2.9 & 3.3 & 5.2 & 3.8 & 0.0 \\
\hline \multirow{2}{*}{\multicolumn{6}{|c|}{ Total debt service due }} & 0.0 & 0.0 & 0.0 \\
\hline & & 45.7 & 43.8 & & 38.0 & 40.4 & & \\
\hline Interest & 22.5 & 25.2 & 24.1 & 18.4 & 15.2 & 11.6 & 12.7 & 13.5 \\
\hline Anortization & 22.1 & 20.5 & 19.7 & 23.0 & 22.8 & 28.8 & 31.7 & 28.0 \\
\hline \multicolumn{4}{|l|}{ Ciange in arrears ( - increase) } & & & .13 .3 & -14.8 & 0.0 \\
\hline Debt relief and canceilation & 23.8 & 22.3 & 8.0 & 0.0 & $\begin{array}{r}-30.0 \\
36.5\end{array}$ & 13.3 & 20.5 & 15.7 \\
\hline $\begin{array}{l}\text { Of which } \\
\text { Paris Club }\end{array}$ & 5.6 & 5.9 & 5.7 & & & & & \\
\hline & & 3.9 & & 0.0 & 0.0 & 0.0 & 2.2 & 4.3 \\
\hline
\end{tabular}

Sources: Autunomous Amortization Fund (CAA); and staff estimates. 
Table 36. Benir: Exchange Rate Developments, 1994-2001

\begin{tabular}{|c|c|c|c|c|c|c|c|c|}
\hline & 1994 & 1995 & 1996 & 1997 & 1998 & 1999 & 2000 & 2001 \\
\hline \multicolumn{9}{|l|}{ Exchange rates } \\
\hline \multicolumn{9}{|l|}{$\begin{array}{l}\text { Period averages } \\
\text { CFA francs per U.S. dollar }\end{array}$} \\
\hline CFA francs per U.S. dollar & 555.20 & 499.10 & $\$ 11.60$ & 583.70 & 590.04 & 615.70 & 712.00 & 728.80 \\
\hline CFA francs per SDR & 795.05 & 757.13 & 742.84 & 803.17 & 800.10 & 840.39 & 929.70 & 922.60 \\
\hline \multicolumn{9}{|l|}{$\begin{array}{l}\text { End-of-period } \\
\text { CFA francs per U.S. dollar }\end{array}$} \\
\hline CFA francs per U.S. dollar & 534.60 & 490.00 & 523.70 & 598.81 & 561.20 & 652.95 & 705.00 & 744.30 \\
\hline CRA francs per SDR & 780.44 & 728.38 & 756.22 & 807.94 & 762.34 & 892.58 & 929.80 & 942.20 \\
\hline \multicolumn{9}{|l|}{ Exchange rate indices $(1990=100) 1 /$} \\
\hline CFA franc per U.S. dollar & 203.92 & 183.33 & 187.89 & 214.38 & 216.72 & 226.14 & 261.51 & 267.68 \\
\hline CFA franc per SDR & 215.19 & 204.93 & 201.06 & 217.39 & 216.56 & 227.46 & 251.64 & 249.71 \\
\hline Nominal effective exchange rate $2 /$ & 53.32 & 55.48 & 55.78 & 53.66 & 55.87 & 53.95 & 50.59 & 51.18 \\
\hline Real effective exchange rate $2 / 3 /$ & 67.16 & 77.41 & 79.40 & 77.82 & 83.77 & 80.88 & 77.31 & 79.69 \\
\hline
\end{tabular}

Source: LMF, Information Notice System.

1/ An increase in the index indicates an appreciation of the CFA franc, period averages.

2/ Trade weighted.

3) Deflated by the relative consimet prices. 
Benin: Summary of the Tax System as of April 2002

(Ail amourts in CFA francs)

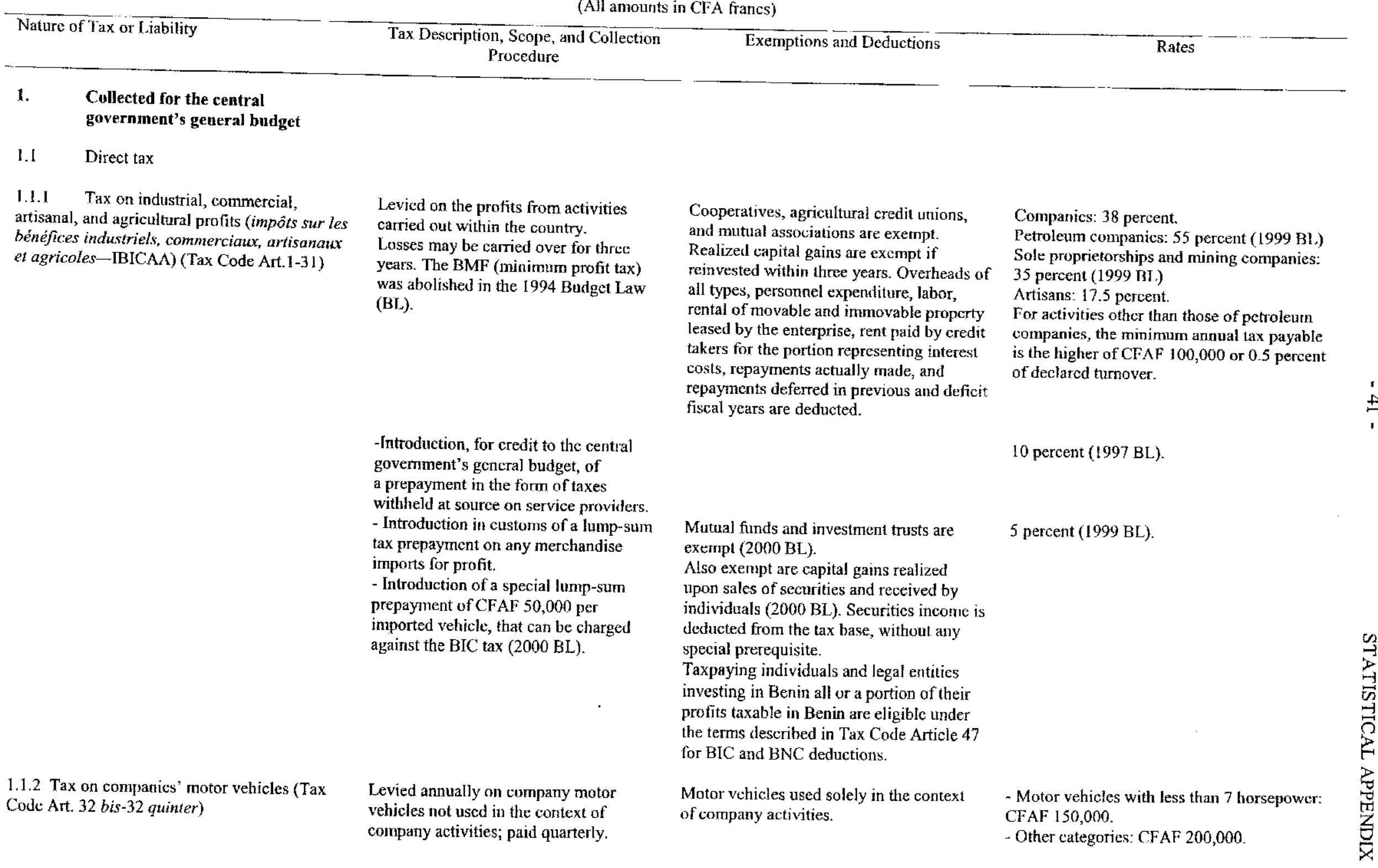


Benin; Summary of the Tax System as of April 2002 (All amounts in CFA francs)

Nature of Tax or Liability
$\begin{aligned} & \text { 1.1.3 Tax on noncommercial profits (impot sur } \\ & \text { les bénéfices non commerciaux--BNC) (Tax }\end{aligned}$

les benefices non commercialu-BNC) (Tax

Code Art. 33-47)

1.1.4 Progressive tax on salaries and wages (impôt progressif sur les traitements et salaries-IPTS) (Tax Code Art. 48-57)

1.1.5 Payroll tax (versement patronal sur salaires-VPS) (Tax Code Art. 58-63, 1996 $B L$ ), payable by employers in place of the former payroll and apprenticeship taxes independent professional activity.

Reasonable expenses necessary for the production of income. No persunal exemptions. Profits received by nonbusiness persons from the sale of company rights totaling no less than CFAF 100,000 are exempt.

Introduction, for credit to the central govermment's general loudget, of a prepayment in the form of taxes witheld at source on service providers.

Withheld monthly from gross salaries, emoluments, wages, earnings, bonuses, overtine compensation, profussional benefits in cash and in kind, and allowances of all types, including for transportation. Payable on gross income without any deduction for pension fund constitution.

Individuals and legal entities paying salaries, emoluments, wages, and incidental compensation are liable for this tax.

Family allowances and social security assistance and wage increases or allowances for social security reasons. For private sector wages, the portion of these allowances or increases exceeding Fund (OBSS) to equally qualificd civi servants of the same grade and marital status is not exempt.

2. Public agercies and offices engaged in

3. Diplomatic representations and
35 percent. However, there is a minimum tax based on minimum profits determined by applying a coefficient ranging from 9 percent to 25 percent, depending on the type of business generating the turnover.

10 percent (1997 BL). the amount payable by the Social Security

CFA

Up to 20

The to 20,000

$20,001-100,000 \quad 15$ percent

$100,001-250,000 \quad 20$ percent

$250,001-500,000 \quad 25$ percent

Over $500,000 \quad 40$ percent

Deductions are allowed in proportion to the number of dependent children, as follows:

$\begin{array}{lr}1 \text { child } & 0 \text { percent } \\ 2 \text { children } & 5 \text { percent } \\ 3 \text { children } & 10 \text { percent } \\ 4 \text { children } & 15 \text { percent } \\ 5 \text { children } & 20 \text { percent } \\ 6 \text { children } & 23 \text { percent }\end{array}$

1. Central and local govemments.

Single rate of 8 percent.

The tax base is the same as for the IPTS.

intcrnational organizations.

4. Taxpayers liable for the single professional tax $(T P U]$. 
Benin: Summary of the Tax System as of April 2002

(All amounts in CFA francs)

Nature of Tax or Liability

(impôt sur les revenus des capitaux mobiliers)

(Tax Code Art. 72-151)

1.1.7 Tax on rental income (taxe immobilière sur les loyers-TIL) (Tax Code Art. 198-204)

1.1.8 General income tax (impôt général sur les revenus-IGR) (Tax Code Art. 152-186)
Tax Description, Scope, and Collection Procedure
Exemptions and Deductions Rates
Withheld at source on dividends, interest, attendance fees, and other forms of distributions of corporate profits to individuals and legal entities, as well as on any sums or securities allocated at any time whatever to partners and

shareholders for any reason other than repayment of their contributions and any hidden distributions whatever

Levied on gross rental income received by individuals and companies.

Levied annually on total net persona income from all sources, excluding salaries and wages.
- Interest on sums entered on savings bank books;

- Interest and any other proceeds of accounts held at credit institutions in the name of the national savings bank; - Interest on investment loans (excluding any commercial loans granted by the banks); and

- Income from obligations received by entities residing outside the West African Economic and Moneixry Union (WAEMU) zone.

None.

The following deductions are allowed on rental income: the schedular tax maintenance costs, and interest. A 30 percent deduction is also allowed for managenent, insurance, and depreciation custs. Personal deductions for dependents are allowed as a percentage of taxable income, ranging from 10 percent for a mlarried taxpayer with no children to 40 percent for a married person with six or more children,

The tax is increased by 30 percent for single, widowed, and divorced taxpayers without dependent children. Income of up to CFAF 100,000 per year is excmpt. Income from bonds received by persons residing outside the WAEMU zone is exempt.
On incone from claims: 15 percent of the interest on deposits and guarantees.

On income from securities (limpôt sur le revenu des valeurs mobilières-IRVM): 18 percent.

The following reductions apply (2000 BL):

- For share proceeds: 10 percent;

- For bond income: 13 percent;

- For lots and redemption premiums paid to creditors and bondholders: 15 percent.

Monthly rental income brackets:

Up to CFAF 50,000: 10 percent; and Over CF $A F$ 50,000: 20 percent.

The general income tax consists of two levies:

(a) a tixed levy ranging from $\mathrm{CFAF} 2,000$ to CFAF 15,000 based on income level and with no reference to family obligations; and (b) a progressive levy based on the following scale:

Up to 100,000

$100,001 \sim 600,000$

$600,001-800,000$

$800,001-1,600,000$

$1,600,001-2,500,000$

$2,500,001-3,500,000$

$3,500,001-4,500,000$

$4,500,001-5,500,000$

Over $5,500,000$

Exempt
6 percent
15 percent
20 percent
25 percent
30 percent
40 percent
50 percent
60 percent


Benin: Summary of the Tax Systom as of April 2002

(All amounts in CFA francs)

\begin{tabular}{|c|c|c|c|}
\hline Nature of Tax or Liability & $\begin{array}{l}\text { Tax Description, Scope, and Collection } \\
\text { Procedure }\end{array}$ & Exemptions and Deductions & Rates \\
\hline
\end{tabular}

1.1.9 Land tax (taxe foncière unique) (Tax Code Art.1084-8)

1.1.10 Professional tax (taxe professionnelle unique-TPU) (Tax Code Art. 1084-9 to 1084-16)

\subsection{Indirect faxes}

1.2.1 Value-added tax (VAT)

(Tax Code Art. 219-272)
Levied on the real rental value of taxable property in areas with an Utban Land Register (RFL), where it replaces the FB, the FNB, the TII, and the land IGR.

Levied on professional rental values. Applicable to small enterprises with tumover of less than:

- CFAF 40 million for purchases and resales;

- CFAF 15 million for services.

Applicable in areas with an RFU.

Levied on the proceeds of sales of goods and services, as well as production and importing.

Applicable to entities with a turnover of more than:

- CFAF 40 million for those engaged in trading and similar activities; and - CFAF 15 million for those in wher activitics.

May be applicable, by taxpayer option,

- sales and services effected by entities with an annual turnover below the limits set by order of the Minister responsible for Finance; and
Properties belonging to the central and local governments, etc. are exempt. There is a temporary 5-year exemption for cxclusively residential buildings.

- Central govemment activities:

- Legal entities;

- Taxpayers liable for the BNC tax;

Pharmacists;

- Construction and public works enterprises; and

- Individuals engaging in a profession for which the specifications require full accounting.

- Proceeds shown in Annex 1 of the 2000 Budget Law Ordinance

- Medical consultations, health care, and any medical services, including the

transportation of injured persons, as well as services provided during stays in hospitals, clinics, and other similar institutions,

excluding care provided by veterinarians;

- Composition, printing, and sales of newspapers and periodicals, excluding advertising income;

- Sales made by nonprofit associations; - Public or private passenger transport; - Postage stamps, revenue stamps, and other similar assets:
- For developed property: 6 percent

- For developed rental property: 12 percent, of which 6 percent is for the national budget - For undeveloped property: 5 petcent However, by deliberation of the elected Representatives, the rates can be reduced or raised by up to two points.

-6 percent of the professional rental value. However, this rate may be reduced or raised by up to two points, by deliberation of the elected Representatives:

-6 percent of the tax base in additional contributions by enterprises with a tumover below the limits set by order of the Minister Responsible for Finance, in place of the $\mathrm{BIC}$ tax, the IGR, and the VI'S.

\section{Single rate of 18 percent.}

-Zero for exports 
Benir: Summary of the Tax System ds of Aprit 2002

(All amounts in CFA francs)

\begin{tabular}{|c|c|c|c|}
\hline Nature of Tax or Liability & $\begin{array}{c}\text { Tax Description, Scope, and Collection } \\
\text { Procedure }\end{array}$ & Exemptions and Deductions & $\overline{\text { Rates }}$ \\
\hline . & - public passenger transport opcrations. & $\begin{array}{l}\text { - Banking and financial operations subject } \\
\text { to the tax on financial activities (TAF); } \\
\text { - Operations subject to the formality of } \\
\text { registration; } \\
\text { - Sales of original works of art by their } \\
\text { authors; and } \\
\text {-Tax-on-tax deductions. } \\
\text { In production and processing operations, } \\
\text { wholesale trade imports, deductions are } \\
\text { allowed of taxes paid upstream on goods, } \\
\text { materials, and equipment that are inputs to } \\
\text { taxable activities. }\end{array}$ & \\
\hline $\begin{array}{l}\text { 1.2.2 Tax on financial activitics (Taxe sur les } \\
\text { activités financières-TAF) (Tax Code Art. 293- } \\
1 \text { to 293-5) }\end{array}$ & $\begin{array}{l}\text { Levied on operations carried out by } \\
\text { banks and other financial institutions, } \\
\text { excluding those not specifically related } \\
\text { to trade in securities and moncy. }\end{array}$ & $\begin{array}{l}\text { - BCEAO operations; } \\
\text { - Operations of lending and credits to the } \\
\text { public treasury and local governments; } \\
\text { - Operations of credits, loans, advances, } \\
\text { deposits on account, commitments, and } \\
\text { similar operations carried out between } \\
\text { banks and financial institutions, whether or } \\
\text { not installed in the Republic of Benin; and } \\
\text { - Operations not specifically related to } \\
\text { trade in securities and money and that are } \\
\text { liable for VAT, such as: sales, rentals, } \\
\text { leasing, studies and consultations, transfers } \\
\text { of fees, invoicing, and similar operations; } \\
\text { - Operations of banks lending to } \\
\text { enterpriscs engaged in the construction of } \\
\text { low-cnst housing. }\end{array}$ & $\begin{array}{l}10 \text { percent on the value before tax of } \\
\text { services, commissions, and interest income. }\end{array}$ \\
\hline $\begin{array}{l}\text { 1.2.3 Insurance tax (taxe unique sur les contrats } \\
\text { d'assurance) (Tax Code Art. 913-[9]34) }\end{array}$ & Levied annually on insurance premiums. & $\begin{array}{l}\text { Insurance contracts concluded by } \\
\text { agricultural mutual insurance companies or } \\
\text { funds are exempt, as well as health and life } \\
\text { insurance contracts, instruments } \\
\text { concerning approved fraternal associations }\end{array}$ & $\begin{array}{l}\text { Firc: } 20.0 \text { percent. } \\
\text { Automobiles and niscellaneous risks: } \\
\text { 10 percent. } \\
\text { Export eredit guarantees: } 0.25 \text { percent. } \\
\text { Tiansportation insurance: } 5 \text { percent. }\end{array}$ \\
\hline
\end{tabular}


Benin: Summary of the Tax System as of April 2002

$$
\text { (All amounts in } \mathrm{CF}^{\mathrm{A}} \mathrm{A} \text { francs) }
$$

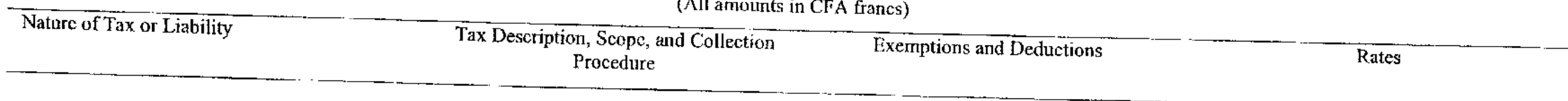

1.2.4 Tax on petroleum products (Tax Code Art. 249 bis- 256 bis $)$

1.2.5 Tax on tobacco and cigarettes (Tax Code, new Art. 257 bis-260 bis

1.2.6 Tax on beverages (Tax Code, new Art. 261 bis-268 bis

1.2.7 Tax on wheat flour (taxe unique sur lo farine de blé) (Tax Code, new Art. 266 bis-270 bis)

\subsubsection{Tax on perfumes and cosmetics (Tax} Code, new Art. 271 bis-27.5 bis $)$

and unions, and craft unions.

Levied on all sales of peroleum products - "Products already taxed; and in Benin (first transfer).

Levied on imported and locally manufactured tobacco and cigatettes.

\section{Levied on:}

- sales of locally manufactured

beverages; and

- imports of beverages into Betin.

- Noncarbonated water;

- Beverages already taxed; and

- Exports.

Levied on locally milled and imported wheat flour.

- Wheat flour already taxed; and

- Exports.

Products already taxed; and - Exports.

- Tohacco and cigarettes already taxed; and - Exports.

Levied on perfumes and cosmetics whether locally manufactured or imported.

Oil and grease: 2 percent.

Regular and super gasoline: 11.16 percent (1998 BL).

Petroleum and diesel oil: 0.5 percent. Applied to the selling price before tax and excluding retailer margins.

\section{8 percent applied to:}

- the customs value of imports, plus duties and taxes collected upon entry, excluding VAT; and

- local production, on the producer price, excluding VAT.

- For nonalcoholic beverages: 3 percent; and - For alcoholic beverages: 8 percent.

Applied to:

- imports at customs value, plus duties and taxes collected upon entry, excluding VAT and

- local production, on the producer price, excluding VAT:

1 percent applicable $t o$ :

- imports at customs value, plus duties and taxes collected upon entry, excluding VAT. and

- local production, on the producer price, excluding V $\mathrm{AT}$.

5 percent applicable to:

- imports at customs value, plus duties and taxes collected upon entry, excluding VAT; and

- local production, on the producer price, 
Benin: Summary of the Tax System as of April 2002

(All amounts in CF $\Lambda$ frartes)

Nature of Tax or Liability
new Art. 276-280)

1.2.10 Tax on gambling (Tax Code Art. 293-6 to Art. 193-8)

1.2.11 Road transportation tax (taxe unique sur les transports routiers)

(Decree 107/DC/SGM/DGID/DLC of

March 14, 2002 amending Art. 6 of

Decree 166/MFE/DC/SGM/DGID/DLC of

March 14, 2001)

1.2.12 Radio and TV tax (taxe radiophonique et télévisuelle) (Tax Code Art. 290-293)
Levied annually on the use of radio and television sets on behalf of the radio and television broadcasting office of Benin.

None.

Vehicles with less than four (4) wheels used for public passenger or merchandise transport are exempt.

- Cooking oils and fats already taxed; and - Exports.

Gambling subject to VAT.

Levied on all gambling, cxcluding those

Levied annually on vehicles used for public passenger or merchandise transport.

- Conciliation minutes ;

- Unnamed instruments;

- Instruments on corporate dissolution excluding VAT.

1 percent applicable to:

- imports at customs value, plus duties and taxes collected upon entry, excluding VAT; and

- local production, on the producer price, excluding VAT.

5 percent applicable to the selling price of tickets for the various games provided for the public by National Lottery of Benin (LNR).

(1) Vehicles for public passenger transport: 0 to 9 seats: CFAF 38,000;

10 to 20 seats: CF $\wedge F 57,000$;

More than 20 seats: CFAF 86,800.

(2) Vehicles for public merchandise transport:

0 to 2.5 metric tons: CFAF 49,500 ;

2.6 to 5 metric tons: CFAF 57,000 ;

5.01 to 10,000 metric tons: CFAF 86,800 ;

Over 10,000 metric: CFAF 136,400.

Arnual tax of CFAF 500 per radio set owned by the taxpayer.

CFAF 700 for two radio sets.

CFAF 900 for threc radio sets.

$C F \Lambda F 1,200$ for more than three radio sets.

CFAF 3,000 per television set.

1.3 Registration and stamp duty

1.3.1 Registration duty (droits

d'enregistrement) (Tax Code Art. 541-586)
Fixed duty of CFAF 500.

Fixed duty of CFAF 1,000 . 
Benin: Summary of the Tax System as of April 2002

(All amounts in CF $\wedge$ tranes)

\begin{tabular}{|c|c|c|c|}
\hline Nature of Tax or Liability & $\begin{array}{c}\text { Tax Description, Scope, and Collection } \\
\text { Procedurc }\end{array}$ & Exemptions and Deductions & Rates \\
\hline & $\begin{array}{l}\text { without transfer of movable or } \\
\text { immovable goods; } \\
\text { - Instruments mentioned in Art. 543-544; } \\
\text { - Instruments mentioned in Art. 545; } \\
\text { - Instruments mentioned in Art. 547; } \\
\text { - First instance and Appeals Court } \\
\text { divorce judgments and decrees; } \\
\text { - Overdrafts granted to business persons } \\
\text { and cash facilitics; } \\
\text { - Consolidated overdrafts, term credits, } \\
\text { and any signature commitments, } \\
\text { including security deposits, } \\
\text { endorsements, documentary credits, and } \\
\text { any special assistance; and } \\
\text {-Property transfcrs, leases, capital } \\
\text { increases, company mergers, and other } \\
\text { transactions, etc. }\end{array}$ & $\begin{array}{l}\text { Clains tradable on the money market. } \\
\text { Furniture leasing contracts. }\end{array}$ & $\begin{array}{l}\text { Fixed duty of CFAF } 2,000 . \\
\text { Fixed duty of CFAF } 4,000 \text {. } \\
\text { Fixed duty of CFAF } 4,000 \text { and CFAF } 7,000 \text {. } \\
\text { Fixed duty of CFAF } 6,000 \text { ( } 2000 \text { BL). } \\
0.25 \text { percent with a minimum of CFAF } 5,000 \\
\text { (1999 BL). } \\
\text { Fixed duty of CFAF } 6,000 \text {. }\end{array}$ \\
\hline $\begin{array}{l}\text { 1.3.2 Inheritance and gift duty (droits sur les } \\
\text { mutations a titre gratuit) (Taxe Code Art. 593- } \\
612 \text { ) }\end{array}$ & $\begin{array}{l}\text { Levied on the value of property } \\
\text { transferred free of charge. }\end{array}$ & $\begin{array}{l}\text { Transfers to the heirs of military personnel } \\
\text { and civilians killed on duty ate exempt. A } \\
\text { CFAF } 6 \text { million deduction is allowed for a } \\
\text { spouse and for each child (up to six). } \\
\text { There is a } 25 \text { percent deduction for } \\
\text { transfcrs under a marriage contract. }\end{array}$ & $\begin{array}{l}\text { Set by bracket (on the net value of the } \\
\text { property) according to the degree of kinship } \\
\text { of recipients, ranging betwcen } 3 \text { percent and } \\
45 \text { percent. }\end{array}$ \\
\hline $\begin{array}{l}\text { 1.3.3 Mortgage duty (droit d'hypothèque) Tax } \\
\text { Code Art. 955-961) }\end{array}$ & $\begin{array}{l}\text { - Registration } \\
\text { - Insertion of a deed transferring or } \\
\text { extinguishing a real right. }\end{array}$ & None. & $\begin{array}{l}\text { - For a parcel of land valued at less than } \\
\text { CFAF I million: CFAF 7,000; and } \\
\text { - For a parcel of land valued at more than } \\
\text { CFAF } 1 \text { million: } 7.5 \text { per thousand plus a fee } \\
\text { of CFAF } 200 \text {. } \\
\text { - On sums mentioned in the deed or, where } \\
\text { applicable, on an estimate provided by the } \\
\text { parties: } 3 \text { per thousand. }\end{array}$ \\
\hline 1.3.4 Stamp duty (draits de timbre) & Levied on: & None. & -Stamp duty of CFAF 50 to CFAF 1,200 \\
\hline
\end{tabular}


Benin: Summary of the Tax System as of April 2002

(All amounts in CF $\Lambda$ francs)

\begin{tabular}{|c|c|c|c|}
\hline Nature of Tax or Liability & $\begin{array}{c}\text { Tax Description, Scope, and Collection } \\
\text { Procedure }\end{array}$ & Exemptions and Deductions & Rates \\
\hline Tax Code Art. 613-777) & $\begin{array}{l}\text { - stamped paper, } \\
\text { - commercial instruments, } \\
\text { - transportation contracts, } \\
\text { - sea transport, and } \\
\text { - bills of lading. }\end{array}$ & & $\begin{array}{l}\text { - Proportional duty of I per thousand ( } 1999 \\
\text { BL); and } \\
\text { - Fixed duty, depending on the amount, on } \\
\text { receipts of: } \\
\text { Up to CFAF 100: none; } \\
\text { CFAF 101-1,000: } 10 \text { percent; } \\
\text { CFAF 1,001-5,000: } 25 \text { percent; } \\
\text { CFAF 5,001-10,000: } 50 \text { percent; } \\
\text { CFAl } 10,001-50,000: 100 \text { percent; } \\
\text { Over CFAF } 50,000 \text { and per bracket of } \\
\text { CFAF } 50,000: 30 \text { percent; } \\
\text { - Fixed duty of CFAF 2,500 (1998 BL). }\end{array}$ \\
\hline \multicolumn{4}{|l|}{ 1.4 Duty and taxes collected by customs } \\
\hline 1.4.1 Customs duty & $\begin{array}{l}\text { Levied on the value of atl imports } \\
\text { intended for the local market. }\end{array}$ & $\begin{array}{l}\text { - Grants to the central and local } \\
\text { governments; } \\
\text { - Diplomatic and consular offices; } \\
\text { - Equipment and capital goods, arms and } \\
\text { ammunition for customs, the police, water } \\
\text { and forests, the army, anul the gendarmerie; } \\
\text { - Grants of objects and articles for the } \\
\text { practice of religion; } \\
\text { - Investment Code; } \\
\text { - Imports of raw materials by local } \\
\text { imdustries; } \\
\text { - Personal ubjects and effects; } \\
\text { - Mailings for the Red Cross, the Red } \\
\text { Crescent, and aid agencies that have signed } \\
\text { an agreement with the government; } \\
\text { - Medical samples; and } \\
\text { - Funeral objects. }\end{array}$ & $\begin{array}{l}0,5,10, \text { and } 20 \text { percent (see Art. } 7 \text { of } \\
\text { Regulation } 02 / 97 / \text { CMUEMOA of } 11 / 28 / 97 \text { ). }\end{array}$ \\
\hline $\begin{array}{l}\text { 1.4.2 Community solidarity levy (Prélèvement } \\
\text { communautaire de solidarité-PCS) }\end{array}$ & $\begin{array}{l}\text { Levied ad valorem on merchandise } \\
\text { imported from third countries by } \\
\text { WAEMU member countries and }\end{array}$ & $\begin{array}{l}\text { - Merchandise in transit; } \\
\text { - Merchandise in warehouses; } \\
\text { - Goods acquired under external financing }\end{array}$ & $\begin{array}{l}1 \text { percent (see supplementary Decree 04/96 } \\
\text { of May } 10,1996 \text { ). }\end{array}$ \\
\hline
\end{tabular}


Benin: Summary of the Tax System as of April 2002

(All amounts in CFA fancs)

\begin{tabular}{|c|c|c|c|}
\hline Nature of Tax or Liability & $\begin{array}{c}\text { Tax Description, Scope, and Collection } \\
\text { Procedure }\end{array}$ & Exemptions and Deductions & Ratcs \\
\hline & marketed. & $\begin{array}{l}\text { contracts subject to an exemption clause; } \\
\text { - Assistance and grants to the central } \\
\text { government and charities; } \\
\text { - Merchindise on which the PCS was paid } \\
\text { under previous artangements; } \\
\text { - Goods imported by enterprises operating } \\
\text { under a stabilized tax system; } \\
\text { - Goods with duty-free diplomatic status; } \\
\text { and } \\
\text { - Petroleum products. }\end{array}$ & \\
\hline $\begin{array}{l}\text { 1.4.3 Statistical tax } \\
\text { (Redevance statistique-RS) }\end{array}$ & $\begin{array}{l}\text { Levied ad valorem on all merchandise } \\
\text { imported: } \\
\text { - for the local market; } \\
\text { - in transit; } \\
\text { - for reexport. }\end{array}$ & $\begin{array}{l}\text { - Diplomatic and consular exemptions; } \\
\text { - Goods acquired under extemal financing } \\
\text { agreements subject to an explicit } \\
\text { exemption clause; and } \\
\text { - Merchandise in transit toward landlocked } \\
\text { countries. }\end{array}$ & $\begin{array}{l}\text { l percent (see Regulation } \\
02 / 2000 / \mathrm{CM} / \mathrm{T} \text { JMOA of June } 29,2000 \text { ). }\end{array}$ \\
\hline 1.4.4 Customs stamp (timbre douanier) & Levied on all customs operations. & $\begin{array}{l}\text { Whenever entry duties or taxes are } \\
\text { payable. }\end{array}$ & $\begin{array}{l}\text { - } 4 \text { percent of the RS; and } \\
\text { - } 4 \text { percent of the exit tax, where applicable. } \\
\text { - Included in VAT. }\end{array}$ \\
\hline 1.4.5 Highway maintenance tax (taxe de voirie) & $\begin{array}{l}\text { Levied ad valorem on all imports and } \\
\text { exports. }\end{array}$ & $\begin{array}{l}\text { - Merchandise transported by rail; and } \\
\text { - Postal packages. }\end{array}$ & $\begin{array}{l}-0.15 \text { percent for marketing operations; and } \\
-0.85 \text { percent for all other operations. }\end{array}$ \\
\hline 1.4.6 Value-added tax (VAT) & $\begin{array}{l}\text { Levied ad valorem on imports for } \\
\text { conunercial purposes. }\end{array}$ & $\begin{array}{l}\text { - Pharmaceuticals and condoms; } \\
\text { - Books and teaching materials; } \\
\text { - Milk; } \\
\text {-Secd products; } \\
\text { - Reproductive animals; } \\
\text { - Cocoa beans; } \\
\text { - Animal food producls; and } \\
\text { - Electric power. }\end{array}$ & 18 percent. \\
\hline $\begin{array}{l}\text { 1.4.7 Information technology tax (redevance } \\
\text { informatique) }\end{array}$ & $\begin{array}{l}\text { Specially levied on each written } \\
\text { declaration. }\end{array}$ & Marketing operations. & - CFAF 2,000 per declaration. \\
\hline
\end{tabular}


Benin: Summary of the Tax System as of April 2002

(All amounts in CFA francs)

Nature of Tax or Liability

1.4.9 Television tax (taxe télévisuelle)

1.4.10 Transit tax on merchandise (Taxe de circulation sur les marchandises)

1.4.11 Transit tax on trucks (Taxe de circulation sur camions)

1.4.12 Import tax on temporarily exempt goods (Taxe d'imporiation en franchise temporaire)

1.4.13 Exit tax (Taxe fiscale de sortie)

1.4.14 Guarantee Funds (Fonds de Garantie)

1.4.15 Special reexport tax (taxe spéciale de réexportation-TSR)
Tax Description, Scope, and Collection Procedure
Exemptions and Deductions
- Included in VAT

CFAF 500 each.

5 percent.

Articles not intended for the national market.

television sets.

Special tax on the following merchandise when declared to be in transit:

- Wool and fine hair fabric;

- Synthetic and artificial fiber fabric;

- Bed linen, table linen, and other

articles;

- Cotton fabric

- Tobacco and cigarettes; and

- Alcoholic beverages.

Special tax on foreign trucks transporting more than 1,500 kilograms in

merchandise.

Special tax on foreign passenger vehicles.

Levied ad valorem on exports.

Levied ad valorcm on merchandise as security deposit for interstate transit operations.

Applied ad valorem on a restrictive list of merchandise in Decree 054/ MFE/DC/SGM/DGDDI/DAR of March 1, 2001.
Products not listed in Decree 054 MFE/DC/SGM/DGDDI/DAR of March 1, 2001.
Still valid but no longer applied.

Specific taxes ranging from CFAF 200 to CFAF 1,500 per item.
CFAF 5,000 per truck.

Diplomatic and consular exemptions.

CFAF 5,000 per vehicle.

All products, except cocoa beans, crude Variable petroleurn, and precious metals.

- Diplomatic and consular exemption; and - Vehicles in circulation.

0.25 percent collected for the Chamber of Commerce and Industry of Benin.

8 percent. 
Benin: Summary of the Tax Systen as of April 2002

$$
\text { (All amounts in CFA francs) }
$$

Nature of Tax or Liability

Communautaire-PC)

1.4.17 Presumptive installment payment (Acompte forfaitaire)

\section{Collected for local government budgets}

2.1 Tax on devcloped property (contribution foncière des propriétés bâties-FB) (Tax Code Art. 976-987)

2.2 Tax on undeveloped property (contribution foncière des propriétés non bâties-- FNB) (Tax Code Art. 988-996)

2.3 Land tax (taxe foncière unique) (Tax Code

Tax Description, Scope, and Collection Procedure

Excmptions and Deductions Rates

Collected ad valorem in the Economic Community of West Africa States (ECOWAS) context on all products from third countries.

Levied ad valorem on imports fir commercial purposes.

Levied on the rental value of developed property, and uncultivated land intended for industrial or commercial purposes, including fixed assets (buildings).

Levied on the market valuc of undeveloped property, as calculated by the DGID.

I.evied on the real rental value of taxable
- Equipment for foreign aircraft and boats;

- Merchandise in warchouses;

- Decorations and trophies;

- Assistance to the government and

charities;

- Merchandise on which the PC has already

been paid under previous arrangements:

- Extemally financed public contracts;

- Diplomatic and consular exemptions; and

- Re-exports by local industrics operating

under special tax arrangements.

Noncommercial imports.

5 percent.

The tax is not applied when the amount collected by customs exceeds the BIC

payable by the importer during the year.

Exemptions: public buildings, sport buildings, schools, buildings for public worship, and medical and welfare

facilities.

A five-year exemption is allowed for new buildings.

Deductions: 40 percent for houses atud

50 percent for factories.

Excmption: public propertics and land (up to 5 hectires of land owned by individuals).

A 30-year exemption applies to the recently cultivated, previously forested areas. to the market value of undeveloped property (c.g., 6 percent in Natitingou).

e, depending on the area.

Example:

25 pereent in Ouidah;

20 percent in Lokossa.

Property bclonging to the central and local 
Benin: Sumuary of the Tax System as of April 2002

(All amounts in CFA francs)

\begin{tabular}{|c|c|c|c|}
\hline Nature of Tax or Liability & $\begin{array}{l}\text { Tax Description, Scope, and Collection } \\
\text { Procedure }\end{array}$ & Exemptions and Deductions & Rates \\
\hline Art. $1084-1$ to $1084-8$ ) & $\begin{array}{l}\text { property in areas with an urban land } \\
\text { register (RFU), where it replaces the FB, } \\
\text { the FNB, the TIL, and the land IGR. }\end{array}$ & $\begin{array}{l}\text { governments and to religious, school, and } \\
\text { university establishments. } \\
\text { A five-year temporary exemption applies } \\
\text { to exchusivcly residential builings. }\end{array}$ & $\begin{array}{l}\text { - For developed [rental] property: } 12 \text { percent; } \\
\text { and } \\
\text { - For undeveloped property: } 5 \text { percent. } \\
\text { However, by deliberation of the elected } \\
\text { representatives, the rates can be reduced or } \\
\text { raised by up to } 2 \text { points. }\end{array}$ \\
\hline $\begin{array}{l}2.4 \text { Professional tax (taxe professionnelle } \\
\text { unique-TPU) (Tax Code Art. 1084-9 to 1084- } \\
15\end{array}$ & $\begin{array}{l}\text { Paid by the taxpayers mentioned in } \\
1.1 .10 \text { above, this replaces the business } \\
\text { license (patente) and the liquor license } \\
\text { (licence) in areas with an RFU. }\end{array}$ & $\begin{array}{l}\text { - Central government activities; } \\
\text { - Legal entities; } \\
\text { - Taxpayers liable for the BNC lax; } \\
\text { - Pharmacists; and } \\
\text { - Construction enterprises; etc. }\end{array}$ & $\begin{array}{l}-6 \text { percent of the professional rental value. } \\
\text { However, this rate may be reduced or raised } \\
\text { by up to } 2 \text { points, by deliberation of the } \\
\text { elected representatives; } \\
-6 \text { percent of the tax base in additional } \\
\text { contributions by enterprises with a turnover } \\
\text { below the limits set by order of the Minister } \\
\text { Responsible for Finance, in place of the BIC } \\
\text { tax, the IGR, and the VPS. }\end{array}$ \\
\hline $\begin{array}{l}2.5 \text { Business license tax (contribution des } \\
\text { patentes) (Tax Code Art. } 997-1032 \text { ) }\end{array}$ & $\begin{array}{l}\text { Levied on individuals and corporations } \\
\text { engaged in commercial, industrial, and } \\
\text { professional activities. }\end{array}$ & $\begin{array}{l}\text { Enterprises liable for the TPU. } \\
\text { Public bodies, civil servants, fishermen, } \\
\text { farmers, home-based artisans, and mining } \\
\text { concessionnaires, for their raw materials } \\
\text { mining and sales. } \\
\text { Newly established enterprises are granted } \\
\text { a temporary five-year exemption. }\end{array}$ & $\begin{array}{l}\text { Two levies are applicable: } \\
\text { - Fixed duty based on the type of activity, the } \\
\text { number of employees, and the equipment; } \\
\text { and } \\
\text { - Proportional duty based on the rental value } \\
\text { of professional premises, ranging from } \\
6 \text { percent to } 17 \text { percent. } \\
\text { Supplementary business license for } \\
\text { procurement: } 0.25 \text { percent } \\
\text { Additional centimes are added to these rates. }\end{array}$ \\
\hline $\begin{array}{l}\text { 2.6 Liquor license tax (contribution des } \\
\text { licenses) (Tax Code Art. 1033-1038) }\end{array}$ & $\begin{array}{l}\text { Levied on the same tcrms as the business } \\
\text { licence tax mentioned above in } 2.5 \text { on } \\
\text { enterprises serving alcoholic beverages. }\end{array}$ & Enterprises liable for the TPU. & $\begin{array}{l}\text { Duty calculated on the basis of the enterprise } \\
\text { category, determined by the type of activity } \\
\text { and the type of beverage marketed. } \\
\text { Fixed duty ranges from CFAF } 7,000 \text { to } \\
\text { CFAF } 60,000 \text {. } \\
\text { Additional centimes are added to these rates. }\end{array}$ \\
\hline
\end{tabular}

